\title{
A saturated SSR/DArT linkage map of Musa acuminata addressing genome rearrangements among bananas
}

\author{
Isabelle Hippolyte*1, Frederic Bakry', Marc Seguin², Laetitia Gardes2,3, Ronan Rivallan2, Ange-Marie Risterucci2, \\ Christophe Jenny ${ }^{4}$, Xavier Perrier ${ }^{1}$, Françoise Carreel5, Xavier Argout2, Pietro Piffanelli2,6, Imtiaz A Khan7, \\ Robert NG Miller8, Georgios J Pappas9 , Didier Mbéguié-A-Mbéguié10, Takashi Matsumoto ${ }^{11}$, Veronique De \\ Bernardinis ${ }^{12}$, Eric Huttner ${ }^{13}$, Andrzej Kilian ${ }^{13}$, Franc-Christophe Baurens², Angélique D'Hont ${ }^{2}$, François Cote ${ }^{14}$, \\ Brigitte Courtois² and Jean-Christophe Glaszmann²
}

\begin{abstract}
Background: The genus Musa is a large species complex which includes cultivars at diploid and triploid levels. These sterile and vegetatively propagated cultivars are based on the A genome from Musa acuminata, exclusively for sweet bananas such as Cavendish, or associated with the B genome (Musa balbisiana) in cooking bananas such as Plantain varieties. In M. acuminata cultivars, structural heterozygosity is thought to be one of the main causes of sterility, which is essential for obtaining seedless fruits but hampers breeding. Only partial genetic maps are presently available due to chromosomal rearrangements within the parents of the mapping populations. This causes large segregation distortions inducing pseudo-linkages and difficulties in ordering markers in the linkage groups. The present study aims at producing a saturated linkage map of M. acuminata, taking into account hypotheses on the structural heterozygosity of the parents.

Results: An $F_{1}$ progeny of 180 individuals was obtained from a cross between two genetically distant accessions of $M$. acuminata, 'Borneo' and 'Pisang Lilin' (P. Lilin). Based on the gametic recombination of each parent, two parental maps composed of SSR and DArT markers were established. A significant proportion of the markers $(21.7 \%)$ deviated $(p<$ 0.05 ) from the expected Mendelian ratios. These skewed markers were distributed in different linkage groups for each parent. To solve some complex ordering of the markers on linkage groups, we associated tools such as tree-like graphic representations, recombination frequency statistics and cytogenetical studies to identify structural rearrangements and build parsimonious linkage group order. An illustration of such an approach is given for the P. Lilin parent.

Conclusions: We propose a synthetic map with 11 linkage groups containing 489 markers (167 SSRs and 322 DArTs) covering $1197 \mathrm{cM}$. This first saturated map is proposed as a "reference Musa map" for further analyses. We also propose two complete parental maps with interpretations of structural rearrangements localized on the linkage groups. The structural heterozygosity in P. Lilin is hypothesized to result from a duplication likely accompanied by an inversion on another chromosome. This paper also illustrates a methodological approach, transferable to other species, to investigate the mapping of structural rearrangements and determine their consequences on marker segregation.
\end{abstract}

\section{Background}

The banana (Musa spp.), including sweet and cooking bananas, is the number one tropical fruit, with a global

* Correspondence: isabelle.hippolyte@cirad.fr

${ }^{1}$ CIRAD, UR Multiplication Végétative, Av. Agropolis, 34398 Montpellier Cedex 5, France

Full list of author information is available at the end of the article production exceeding 100 million tons in 2006. It is also a staple food for more than 400 million people [1]. Largely due to technological requirements for transportation and agronomic performances, $45 \%$ of world consumption relies on a single genotype (cv. Cavendish), which is susceptible to the main Musa diseases [2]. It is therefore 
urgent to breed new, disease-resistant genotypes that can be cultivated with less pesticide.

Musa is a Monocot with four known genomes (A, B, S and $\mathrm{T}$ ) and a relatively small genome size of $500-600 \mathrm{Mb}$ in its haploid state. Two species, $M$. acuminata $(2 \mathrm{n}=2 \mathrm{x}=$ $22)$ and $M$. balbisiana genomes $(2 \mathrm{n}=2 \mathrm{x}=22)$ participate to most edible triploid bananas and contain an A and $\mathrm{B}$ genome, respectively.

Musa textilis from Australimusa section $(2 \mathrm{n}=2 \mathrm{x}=20)$ and $M$. schizocarpa $(2 \mathrm{n}=2 \times=22)$, carrying $\mathrm{T}$ and $\mathrm{S}$ genomes respectively, are involved in few edible cultivars[3,4]. Cultivated triploid clones (AAA such as Cavendish, $\mathrm{AAB}$ such as plantain varieties, and $\mathrm{ABB}$ cultivars) are difficult to cross because of sterility, polyploidy, high heterozygosity, interspecificity and low gamete fertility, thus limiting banana improvement [3]. Sterility is generally associated to genome structural heterozygosity. These structural differences likely contribute to crossing barriers within the species. Consequently, the Musa acuminata complex has been divided into seven "translocation groups" [5].

The most widely distributed type is designated the "standard" or "central" group, because of its broad distribution in the M. acuminata species and in other Musa species [5]. In M. acuminata, microcarpa subsp., banksii subsp. and most malaccensis subsp. share this structure. The other six groups (Northern Malayan, Northern 1 and Northern 2, Malayan Highland, Javanese, East African) are defined on the basis of chromosome pairing during meiosis. Within each group, wild accessions share the same chromosome structure and are structural homozygotes, in contrast to most cultivated accessions. In previous characterizations, the inversions were not likened to "translocations", even if chromosome segment inversions was suspected [5]. The fertility of all cultivars is altered by their structural heterozygosity and sterility increases with the number of rearrangements/structural differences [5].

Despite the importance of a well established genetic map to sustain banana genetic improvement at diploid and triploid levels, this tool is presently lacking because of difficulties with Musa in developing a mapping population free of any structural rearrangement. The previous efforts $[6,7]$ highlighted the likely presence of rearrangements but did not provide an interpretation in terms of the structure of the affected chromosomes.

The first mapping experiment with Musa produced a non-saturated genetic map [6], which exhibited 15 linkage groups with 77 markers, among which $36 \%$ significantly deviated from Mendelian segregation $(p<0.05)$. In that study, the $F_{1}$ parent, selfed to generate the segregating progeny, was shown to be heterozygous for two reciprocal translocations. The second map was drawn from 89 individuals coming from a selfed $M$. acuminata diploid "M53". It displayed 11 linkage groups and also distorted markers [8]. The third map featured 14 linkage groups [7]; 59\% of the 120 markers were skewed $(\mathrm{p}<0.05)$ and the $F_{1}$ hybrid used to generate the $F_{2}$ population carried at least two translocations, if not three. Pseudo-linkages could have led to the establishment of oversized linkage groups comprising distorted markers supposed to be involved in the structural rearrangements [9].

A fourth map was to generate a refined $M$. acuminata parental map that could serve as a dense reference Musa genetic map containing the 11 expected linkage groups. Mapping was performed using a F1 population of diploid Musa acuminata genotypes. The female parent was the wild M. acuminata 'Borneo', subsp. microcarpa, supposed to be a structural homozygous. The male parent was the cultivar M. acuminata 'Pisang Lilin', subsp. malaccensis, exhibiting a Northern Malayan/Standard heterozygous chromosomic structure [5,10]. Therefore, the structural heterozygosity of the progeny, named Borli population, should be limited to a unique rearrangement. This work was enabled by combining methodological approaches (DArTs and SSRs) with analytical approaches (Neighbor joining trees) to determine the structure or large chromosomal rearrangements and their location in the genetic maps of the parents of the population.

\section{Results}

\section{Meiotic configuration}

Like many banana cultivars, the male parent $M$. acuminata 'P. Lilin' contained structural chromosome rearrangements, while the wild female parent $M$. acuminata Borneo, is supposed to be free of any.

Meiotic preparations of Borneo and P. Lilin were analyzed. They both displayed some Pollen Mother Cells (PMC) with normal chromosome pairing forming 11 bivalents (Table 1 configuration A) and some PMCs showing some degree of multivalent pairing. Borneo showed one cell displaying one trivalent and one tetravalent (configuration $\mathrm{H}$ ), one cell displaying one pentavalent (Table 1 configuration G) and one cell displaying a hexavalent (Table 1 configuration $\mathrm{F}$ and Figure 1-A). On this basis, we infer that Borneo has at least two structural polymorphisms linking three pairs of chromosomes. This was not expected because Borneo, which is a seeded wild accession with good male and female fertility, was described as structurally homozygous [5].

Meiotic configurations of the P. Lilin parent revealed less complex features. The presence of various cells with only one trivalent (Table 1, configuration C, Figure 1-B) as well as one cell showing an open tetravalent (Table 1, configuration D) led us to tentatively assume one structural polymorphism in P. Lilin. In addition, the bridge observed in one PMC at anaphase-I (Table 1, configuration J) suggested the presence of one chromosome fragment inversion. It is noteworthy that no "closed 
Table 1: Meiotic configurations at metaphase-I and anaphase-I in the parents of the Borli population.

\begin{tabular}{llll}
\hline Metaphase-I configurations & Cell configuration & $\begin{array}{l}\text { Number of PMCs scored } \\
\text { M. acuminata 'Borneo' }\end{array}$ & M. acuminata 'P. Lilin' \\
\hline A & $11 \mathrm{II}$ & 13 & 4 \\
B & $10 \mathrm{II}+2 \mathrm{I}$ & 1 & 3 \\
C & $9 \mathrm{II}+1 \mathrm{II}+1 \mathrm{I}$ & $1^{* *}$ \\
D & $9 \mathrm{II}+1 \mathrm{IV}$ (open) & $1^{*}$ & - \\
E & $8 \mathrm{II}+2 \mathrm{III}$ & 3 & - \\
F & $8 \mathrm{II}+1 \mathrm{VI}$ & 1 & - \\
G & $8 \mathrm{II}+1 \mathrm{I}+1 \mathrm{~V}$ & 1 & - \\
H & $7 \mathrm{II}+1 \mathrm{I}+1 \mathrm{III}+1 \mathrm{IV}$ & 1 & 1 \\
Anaphase-I configurations & & 2 & 17 \\
I & $11 / 11$ chromosomes & - & - \\
J & $10 / 10$ chromosomes +1 bridge & - & - \\
Total no. of cells scored & & 21 & \\
\hline
\end{tabular}

I: monovalent - II: bivalent -- III: trivalent -- IV: tetravalent -- V: pentavalent -- VI: hexavalent.

*: IV open X shape; ** IV open Y shape. PMC: Pollen Mother Cells

tetravalent" was observed in these preparations. Therefore, it is not possible to establish the presence of a true translocation across chromosomes. The meiosis observations on P. Lilin are consistent with previous work $[10,11]$ on this same clone that also drew the conclusion of an inversion.

\section{Marker polymorphism \\ SSR markers}

The SSR marker polymorphism in parents was tested for the 395 primer pairs selected (357 from M. acuminata and M. balbisiana genomic SSRs, and 38 from ESTs). Two hundred and fifty-six primer pairs generated PCR amplicons, among which 181 had polymorphism detected, and exhibited clear and unambiguous singlelocus amplification on the parents.

Of these 395 SSR markers tested, the 29 SSR markers defined on $M$. acuminata "Gobusik" have been extensively used in mapping $[6,12]$ and diversity analysis $[13-$ 15]. The other ones are newly defined. This may explain that $76 \%$ of the former were mapped, while only $43 \%$ of the latter were usable.

Borneo was less heterozygous than P. Lilin. Of the 174 mapped SSR, Borneo displayed 126 heterozygous patterns (72\%) as opposed to 151 for P. Lilin (87\%); 103 of the segregating markers segregated in both parents (59\%), while 23 SSR markers segregated only in Borneo and 48 SSR markers in P. Lilin. Genotyping data are available on GCP registry http://gcpcr.grinfo.net/index.php?app=data sets\&inc $=$ files list.

\section{DArT markers}

The two parents and 92 progenies were hybridized on DArT array. Four hundred and eighty-five markers were found to be polymorphic out of the 11520 present on the array (4\%). Among the 485 markers, 59 could be attributed to a linkage group but were impossible to map (inability to define phases, generation of negative distances and high value of marker mean square contribution ...). Among the 426 DArTs markers that were mapped, 144 (34\%) were contributed by Borneo only, 228 (53\%) were contributed by P. Lilin only, while 54 (13\%) were contributed by both parents. In the reference map, 62 fully identical markers, probably resulting from redundancy, were discarded. Genotyping data are available on the GCP registry http://gcpcr.grinfo.net/index.php?app= datasets\&inc $=$ files list.

\section{Anchorage between parental maps}

The attribution of markers to one of the two parents enabled development of two parental maps. As a first step towards a tentative synthetic map avoiding parent-specific pseudolinkages, the two parental maps were compared at different LOD scores using 133 common markers (79 SSRs and 54 DArTs markers) serving as anchors. The congruence between parental linkage groups was best at LOD 3.5 for Borneo and LOD 5 for P. Lilin (Figure 2). Five consensus linkage groups (i.e. LG 3, LG 5, LG 7, LG 9 and LG 11) were identified on the basis of the full co-linearity of the anchor markers. For the other groups, marker alignments or groupings differed 


\section{A Borneo}
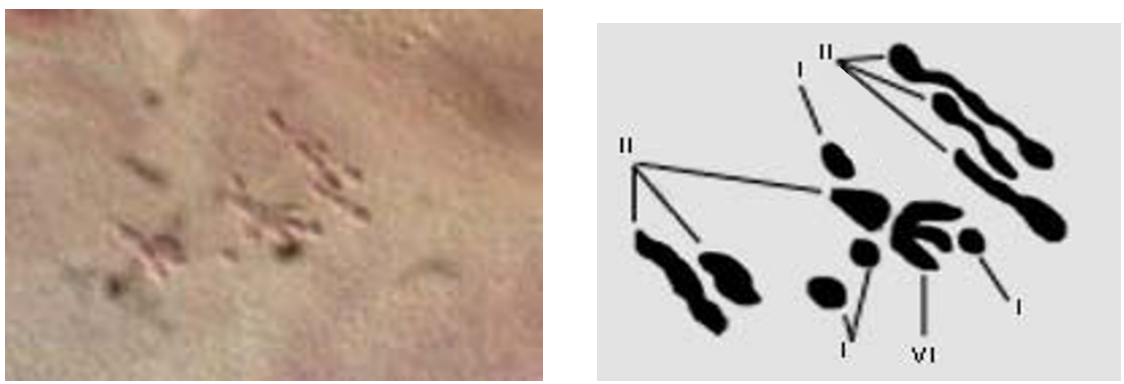

B P. Lilin
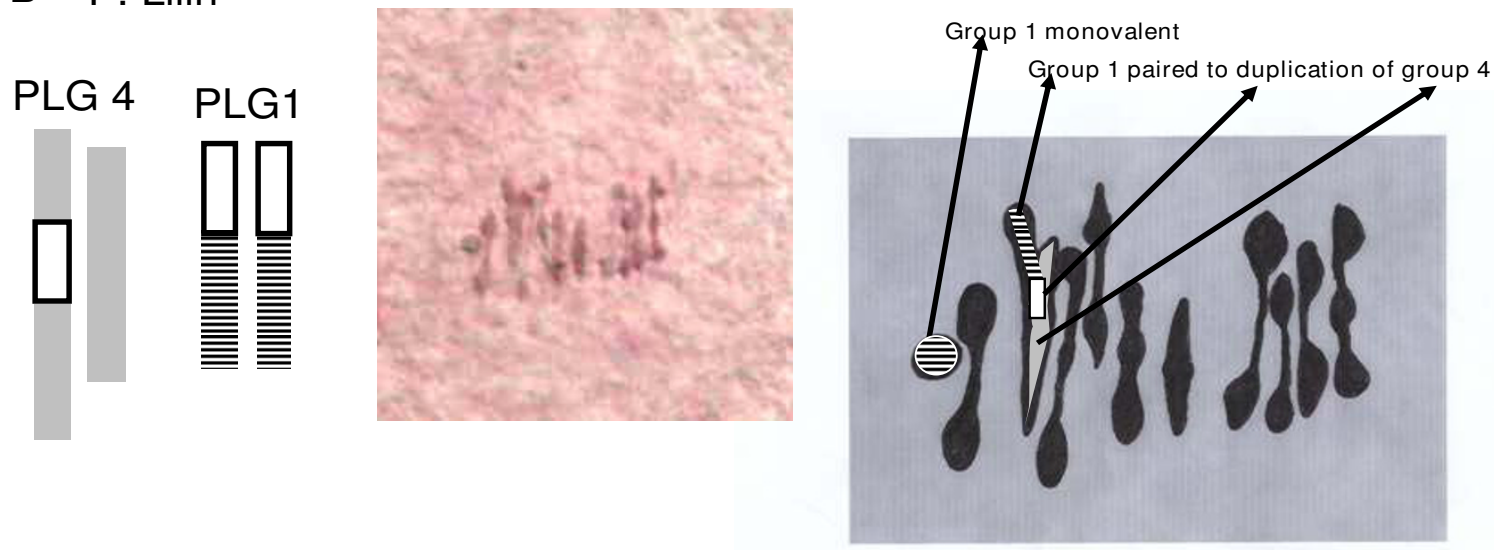

Figure 1 Views of chromosome pairing during meiosis (metaphase I) and their diagrammatic illustration. A - Borneo: the plate represents 4 monovalents (I), 6 bivalents (II) and one hexavalent (VI). The hexavalent is the result of association of three bivalents likely by their distal segments. B P. Lilin: The plate shows 1 monovalent, 9 bivalents and 1 trivalent displaying a Y shape. This pattern suggests a connection point located in the proximal position.

between the parental maps. At LOD 5, the Borneo markers homologous to markers of PLG6 split into two groups. They still split at LOD 3.5, but some of them aggregated with markers homologous to those of PLG 8. The groups BLG1, BLG2 and BLG 4 in the Borneo map built at LOD 3.5 lump into a major group in the P. Lilin map (PLG1+2+4) even up to LOD 8. At LOD 9, the P. Lilin map exhibited 14 linkage groups and the grouping was no longer consistent with the Borneo representation.

The map based on Borneo female parent had 11 linkage groups that were delineated at LOD 3.5 with 261 markers (125 SSRs and 136 DArTs) (Additional file 1). The map spanned about $920 \mathrm{cM}$, with an average marker spacing of $3.8 \mathrm{cM}$. The largest linkage group comprised 59 markers whereas the smallest encompassed 9 markers. Of the 278 segregating markers initially tested, 8 DArTs remained ungrouped and $\mathrm{mMaCir} 120$ was removed.
Regarding the P. Lilin parent, the map obtained at LOD 5 comprised 359 markers of the 379 initially tested (147 SSRs and 212 DArTs), distributed in 9 main linkage groups (PLG) (Additional file 2). The map spanned about $1081 \mathrm{cM}$ with an average marker spacing of about $2.9 \mathrm{cM}$. The markers were not uniformly distributed, one major group (PLG 1+2+4) comprising 113 markers. Sixteen markers remained ungrouped, including 4 SSRs and 12 DArTs. Four more DArT markers (292027, 292284,292234 and 295644) were removed because they disrupted the order of the linkage groups (negative distances; suspect double recombinants).

\section{Segregation distortions}

Twelve percent of the markers deviated from the expected Mendelian ratio ( $x^{2}$ test, significance $p<0.005$ ) on the Borneo female parent (31/269), whereas this percentage reached $24 \%$ with the P. Lilin male parent (89/ 375). So, Borneo exhibited half the rate of highly dis- 


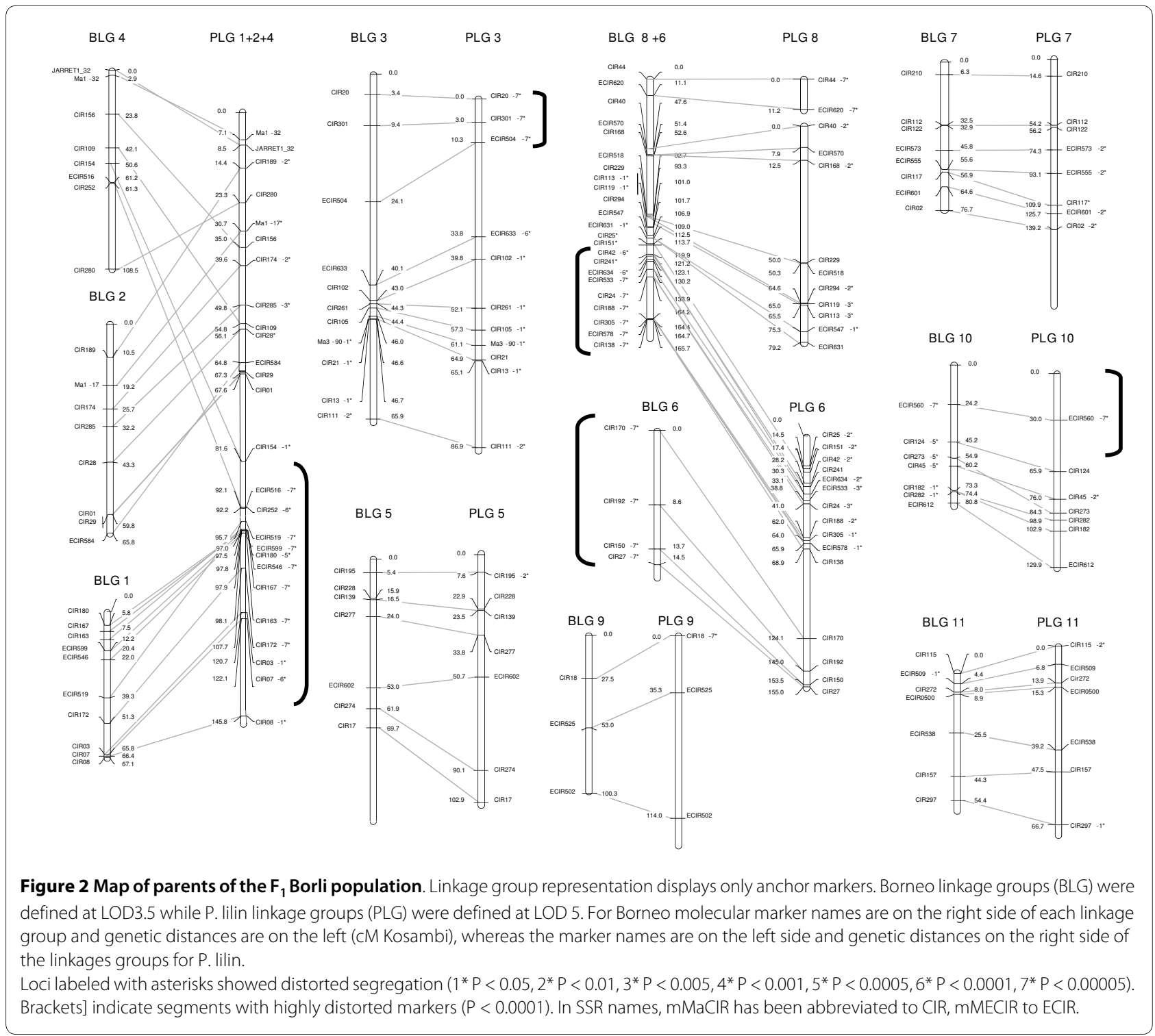

torted markers of P. Lilin. The distortions were of the same order of magnitude for SSR and DArT markers.

Skewed segregations affected different linkage group segments of the parental maps. For example, markers segregated without any distortion on BLG 1, BLG 2 and BLG 4 , whereas half of the markers were highly distorted on PLG $1+2+4$. Similarly, markers on the homologous Borneo group followed Mendelian ratio (1:1) while one segment on PLG 3 showed strongly skewed marker segregations (Cir20 to ECIR633, Figure 2). Conversely, Borneo was affected by highly skewed markers on the segmented BLG 6 while the corresponding loci on P. Lilin exhibited weaker distortions (Figure 2). Among the most highly significant distorted segments (i.e. $\mathrm{p}<0.0005$ ), allelic ratios of the markers varied from 1:2 to $1: 5$ depending on the linkage group and sometimes on the location within a linkage group.
Linkage group tree representations

Adapted tree analyses provide an alternative representation of linkage groups. Trees have been drawn from simulated data of different features of chromosomal rearrangements (Figure 3 and methods). It was applied to all P. Lilin parental linkage groups defined by JoinMap 4 at LOD 5. Figure 4 summarizes the different patterns obtained from the observed data. Most of the P. Lilin linkage groups displayed figures of homologous chromosomes (Figure 4-A) with alignment of markers along the NJ tree similar to that of Figure 3-A, even for PLG 3 (Figure 4-B) which displayed skewed segregations. Two atypical NJ trees were observed for PLG 10 (Figure 4-C) and PLG $1+2+4$ (Figure 4-D).

Concerning PLG 10, we propose that a nested inversion pattern differentiates the two homologous chromosomes (Figure 5). This inversion should fit the observed cytoge- 


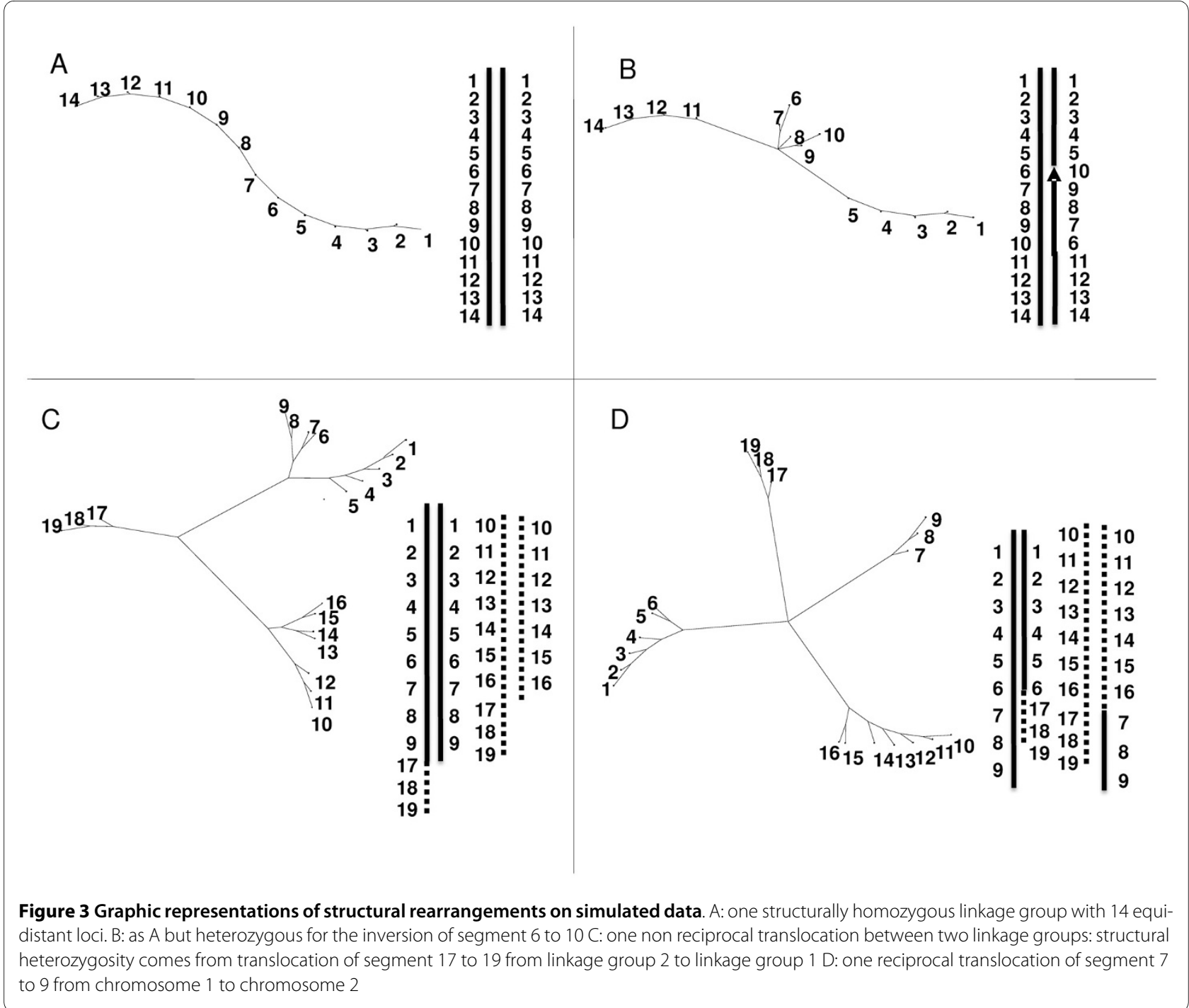

netical inversion features. When this possibility was subjected to NJ tree analysis, we observed a good homology between the observed and simulated trees (Figure 5).

The representation of PLG 1+2+4 (Figure 4-D) is more complex. The homologs of the anchor markers from BLG 1 and BLG 4 are tightly linked, while the homolog markers of BLG 2 are loosely linked to those of BLG 1 and BLG 4. Actually, the aggregation of PLG 2 (Figure 4-D) looked very similar to those of independent groups artificially grouped at LOD 1(Figure 4-E), probably indicating a pseudo-linkage due to skewed markers [16], and PLG 2 is independent from PLG 1 and PLG 4.

In contrast, the pattern of markers from PLG 1 and PLG 4 suggests a "translocation" of markers from PLG 1 into PLG 4 in proximal location. The rearrangement is non-reciprocal as the NJ tree (Figure 4-D) would display three arms instead of four in case of reciprocity as observed in Prunus [17] and derived from a simulation as shown in Figure 3-D. Furthermore it looks like the typical $\mathrm{Y}$ image of Figure 3-C. The best hypothesis would be the existence of a duplication, as suggested by Wilson [11], of a segment of PLG 1 into PLG 4 (Figure 6), but not a true translocation [10] as we neither observed the segregation ratio nor the genotyping profiles expected with a translocation. This hypothesis of segment duplication, associated with lethality of the type of gamete containing the duplicated segment in heterozygous configuration (Figure 6) is also consistent with the observed allelic pattern and allelic frequencies and is in agreement with meiosis configurations (Figure 1-B). The expected Mendelian segregation (i.e. 1:1) observed at the ends of PLG 4 and PLG 1 might result from higher recombination rates in telomeric segments [18] associated with the progressively decreasing rearrangement effect on segregation ratios with increasing distance from the inserted segment. Furthermore, the genetic distance observed between mark- 


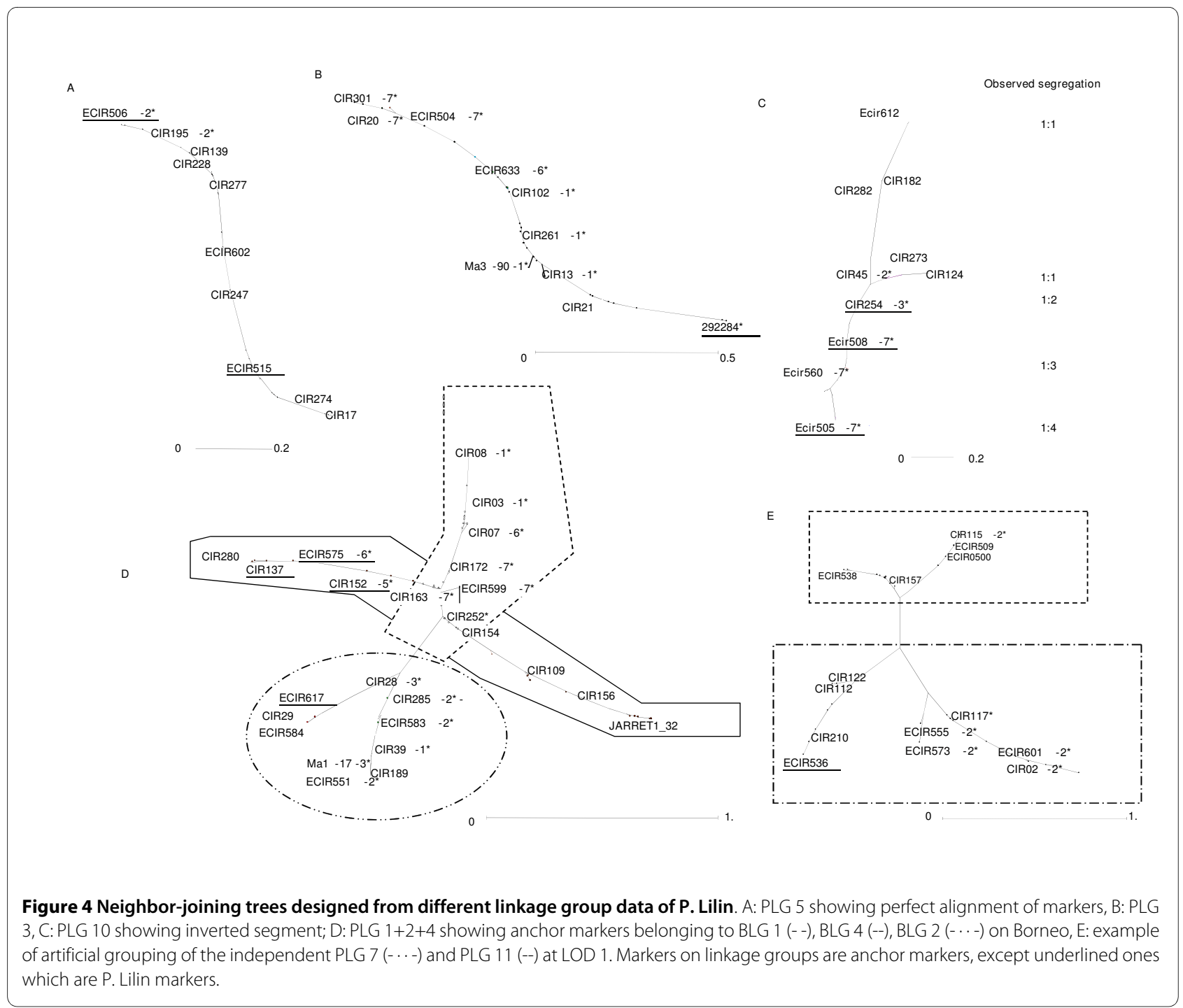

ers within this duplicated segment is low, as found in other studies [19]; [20]. Taking into account this low recombination frequency in the duplicated segment and the increasing frequencies of recombination from the inserted segment to the chromosome ends, the theoretical and observed NJ trees (Figure 6) are very similar.

On PLG 3, we observed highly skewed markers $(\mathrm{P}<$ $0.0005)$ especially at the end of the linkage group, while corresponding markers on the same Borneo group are not distorted (Figure 2). These distortions are located in segments that are collinear when mapping with JoinMap 4 and that also align along the NJ tree representation (Figure 4-B). Therefore we propose as a hypothesis that this region may be subjected to gene selection, meiotic drive or epigenetic effect rather than affected by structural rearrangements.

Overall, based on NJ tree analysis, allele segregations and cytogenetical studies, we propose the presence of two structural rearrangement events for P. Lilin. The first is assumed to be a segment duplication of PLG 1 into PLG 4 (Figure 4-D and Figure 6), instead of a translocation. The second can be a translocation of a PLG 10 segment into itself (Figure 5). Figure 7 presents a putative map of P. Lilin integrating these structural rearrangements.

For Borneo, at LOD 3.5, representations given by the NJ tree seem to indicate a translocated segment from BLG 6 to BLG 8 (Additional file 3). To reproduce the pentavalent or hexavalent pairing features (specific to rearrangement events involving 3 chromosomes) observed on meiosis plates (Figure 1-A), we need to decrease the grouping LOD score down to 2.9. In this case, parts of BLG 6, BLG 7 and BLG 8 are associated. Nevertheless, even in this case, we did not find any chromosome rearrangement model that could explain the very high distortions $(\mathrm{P}<0.0005)$ observed on homolog markers of PLG 


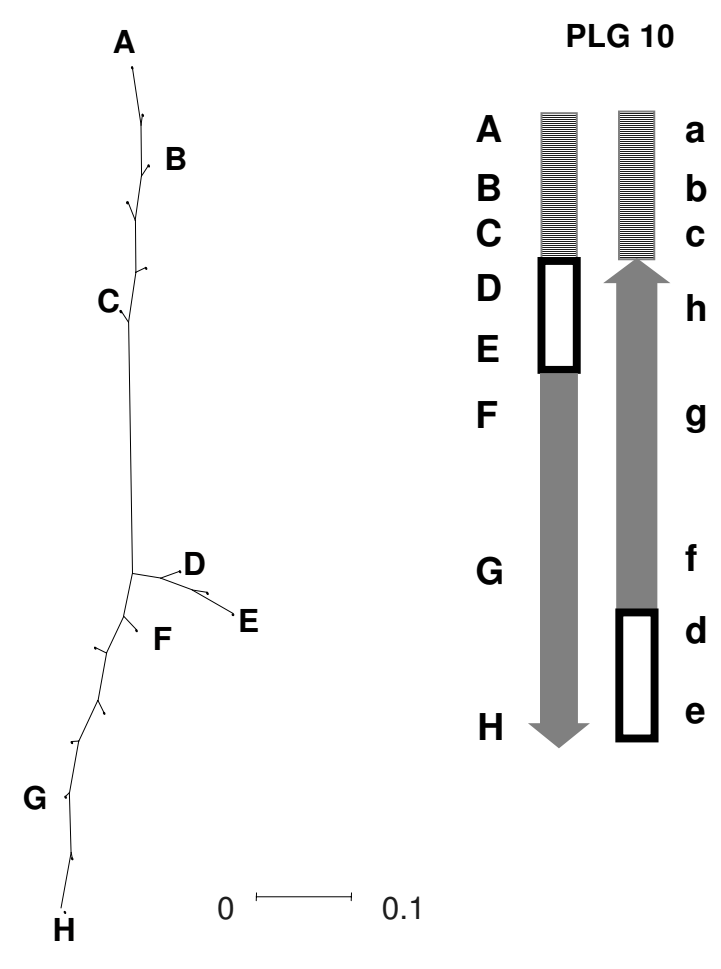

Simulated

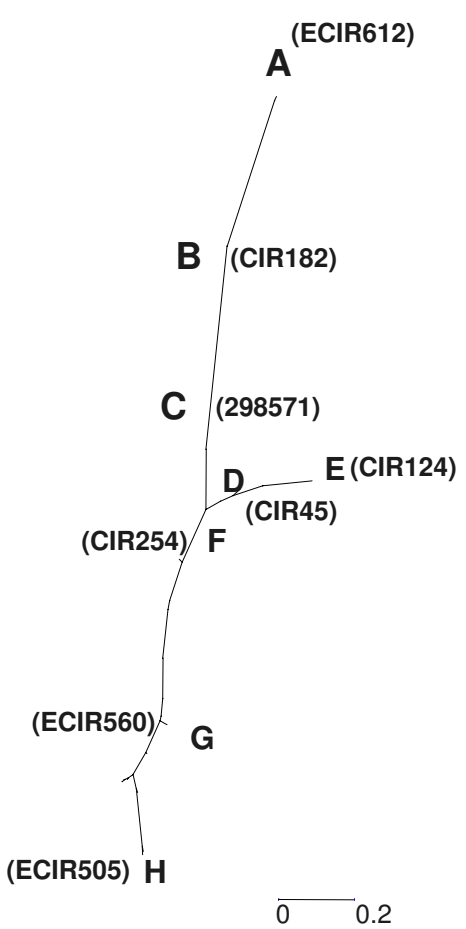

Calculated

Figure 5 Putative rearrangement event on PLG 10. The figure presents the Neighbor-joining tree designed from Kosambi distance calculation, the putative scheme of the rearrangement, and the simulated Neighbor-joining tree obtained with this kind of rearrangement. In SSR names, mMaCIR has been abbreviated to CIR, mMECIR to ECIR.

6, while BLG 7 and BLG 8 did not display any. Furthermore, all homologous markers of PLG 6 only aggregate at LOD 1 . The third group indicated by cytogenetical studies is still not clearly found, nor is the kind of rearrangement that can lead to such a feature.

\section{Synthetic map}

A final synthetic map was constructed at LOD 5 (Figure 8). It was first established from the aligned parental linkage groups described above (i.e. LG 3, LG 5, LG 7, LG 9 and LG 11). For the remaining linkage groups, the grouping and the marker alignments kept as skeleton were chosen from the parent assumed to be free from any structural rearrangement on the considered linkage group. The absence or presence of putative structural rearrangements was assessed with $\mathrm{NJ}$ tree representation and segregation analysis. Priority was given to linkage groups exhibiting a linear NJ tree and Mendelian segregations. A few cases of linear NJ tree with limited distorted segregation were also retained.
From the 426 initial DArT markers, 62 fully identical markers were discarded and 8 DArT markers remained ungrouped. Five SSR markers and 34 DArTs associated with structural rearrangements in one parent were discarded from this reference map because they disrupted its construction (i.e. negative distances, suspect double recombinants, high mean square contribution...). Therefore, some markers present in the parental maps are absent from the reference map. The observed recombination frequency between two markers, one located in the structural rearrangement and one outside, aggregates the results of two different situations: one is linkage, the other one is independency. In practice, the recombination frequency is the mean of recombination of linked markers $(\mathrm{REC}<0.4)$ and of independent markers $(\geq 0.4)$ in the reference map. Consequently, these markers disrupt both the calculation distances between markers and the ordering when compared to data obtained from the non-rearranged parent. 


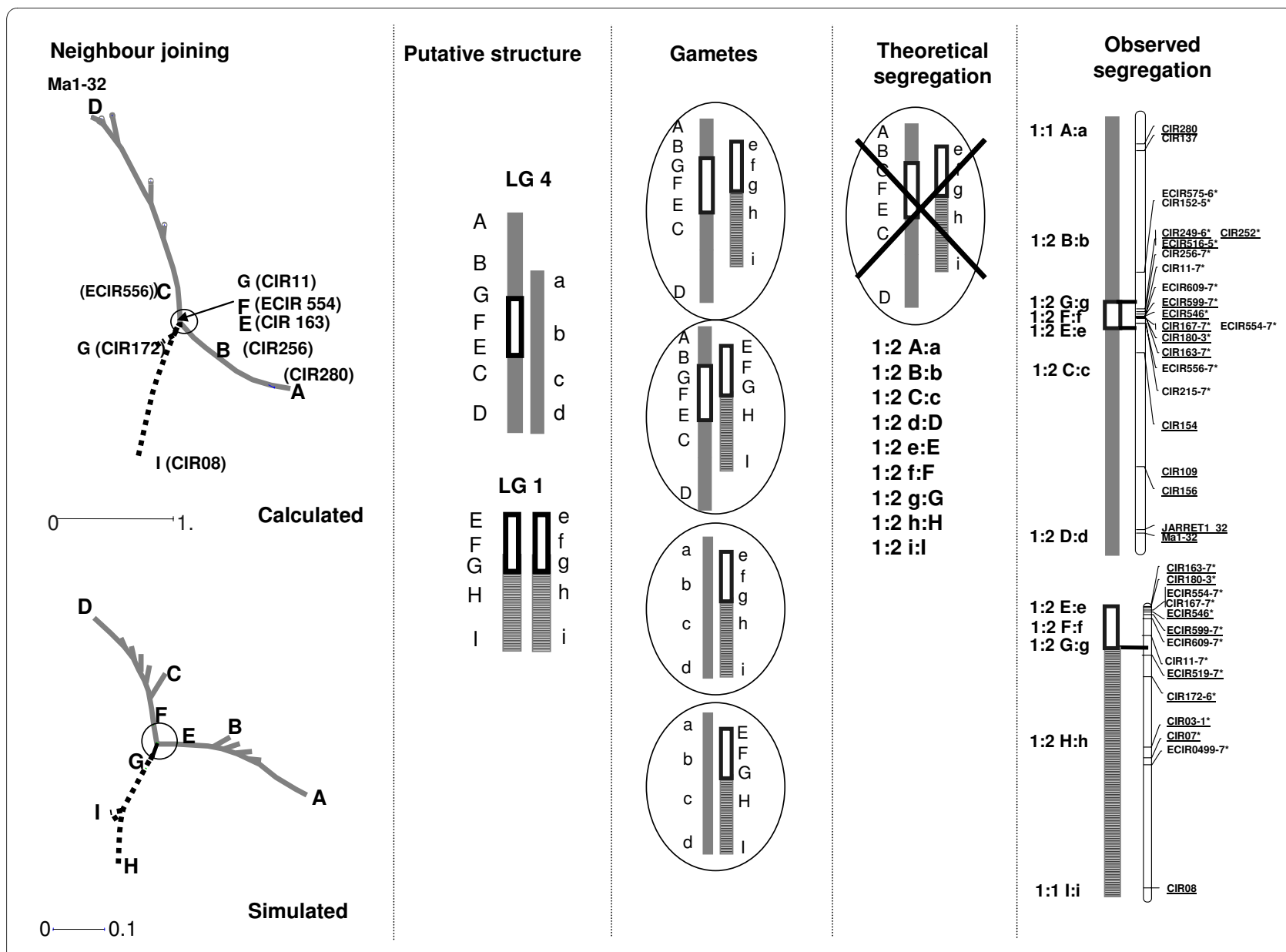

Figure 6 Putative rearrangement event between PLG 4 and PLG 1 in P. Lilin. The observed segregations are given along the linkage groups. Loci labeled with asterisks showed distorted segregation ( $1^{*} P<0.05,2^{*} P<0.01,3^{*} P<0.005,4^{*} P<0.001,5^{*} P<0.0005,6^{*} P<0.0001,7^{*} P<0.00005$ ). Main gametes do not take into account the different allelic combination generated by recombination between homologous segments. Underlined loci are anchor markers. The solid grey line corresponds to PLG 4 and the dotted lines to PLG 1. In SSR names, mMaCIR has been abbreviated to CIR, and $m M E C I R$ to ECIR.

Altogether, this synthetic map includes 489 markers (167 SSRs, 322 DArTs) among which 132 are anchor markers. It is divided into 11 linkage groups covering 1197 cM. The markers are distributed with a mean of 38 markers per linkage group and an average marker spacing of $2.8 \mathrm{cM}$.

\section{Discussion}

This study reports an important effort in marker development and linkage analysis. SSRs provide co-dominant, multi-allelic, locus-specific markers which simplify both the construction of each parental genetic map and the comparison between the two parental maps. DArT provide dominant markers which are generally well-distributed in the genome and very cost-effective [21,22]. They efficiently contribute to map saturation and they constitute an asset that can easily be used for other materials in the future. The main difficulty of our study was the gen- eral occurrence of segregation distortions and the risk of pseudolinkages.

Distortions from expected Mendelian segregation have been observed in both inter-specific and intra-specific derivatives with different magnitudes. They can have multiple origins, including structural rearrangements [6,17,19,23-29].

In the genus Lens, for example, distorted markers were observed in different linkage groups, but only one translocation was detected by cytogenetical studies and pollen viability analysis; its location was defined on the basis of marker locations in different crosses [23]. In Helianthus, [26], reciprocal translocations were described by conjugating observations of abnormal pairing in meiosis, studies on pollen viability and mapping data. The latter revealed an abnormally large linkage group covering close to half of the map. Nevertheless, the causes of the observed segregation distortions often remained unclear. 


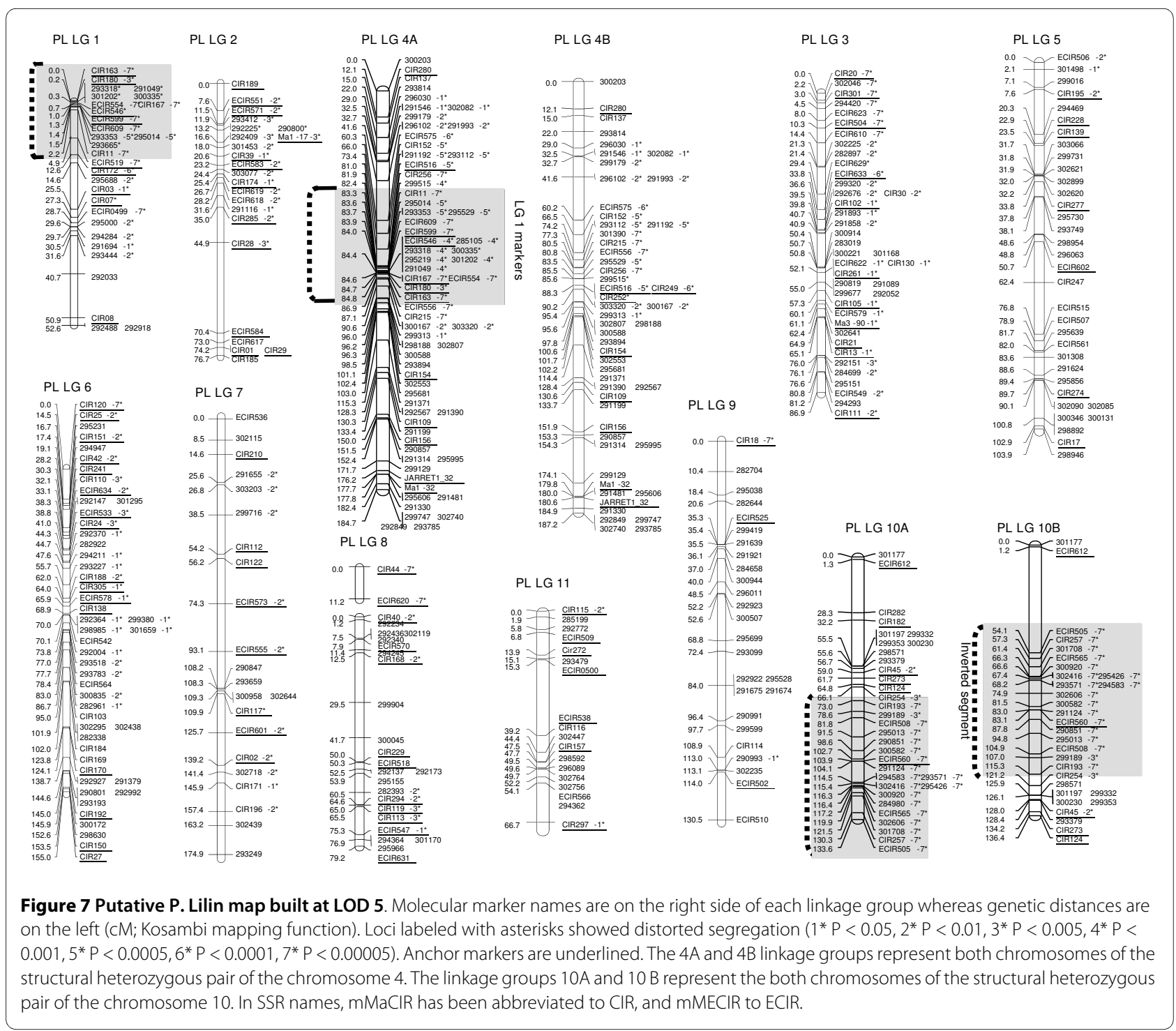

In Prunus inter-specific crosses skewed markers were located on a reciprocal translocation [17]. The hypothesis was validated by studying pollen fertility in the segregating progeny and by cytogenetical observations during meiosis. Most examples show that an array of methods is generally needed to differentiate between the different causes of segregation distortion.

In Musa, earlier studies did not determine the causes of segregation distortion $[6,12]$. Our use of NJ tree representations helped sort between the segregation distortions linked to structural rearrangements and those due to other phenomena such as gene selection, meiotic drive or epigenetic transmission effects. This use led to identification of one likely case of local direct selection on PLG3 and a couple of likely structural rearrangements.

Earlier cytogenetic studies of meiosis in P. Lilin $[10,30]$ described this cultivar as a structural heterozygote featuring one translocation and at least one inversion on the basis of trivalents and bridges. In subsequent observations, Dodds and Simmonds [30] and Shepherd [5] suggested that one of the exchanged segments contains a small sub-terminal inversion, but Wilson [11] stressed that no closed tetravalent was observed and suggested a duplication rather than a translocation. Our interpretation features a duplication between PLG 1 and PLG 4 and an inversion within PLG 10; it is therefore fully in line with earlier inferences.

The structural status of Borneo is less clear. Early cytogenetical studies described Borneo as a structural homozygote [5], in consistency with its full fertility; yet some rearrangements are suspected as well as the presence of a unit with "one of both arms rather short" [5] Our cytogenetic observations suggested that it is heterozygous for at least two rearrangements involving three linkage groups. Some BAC-FISH experiments should be undertaken, as was done for M. acuminata 


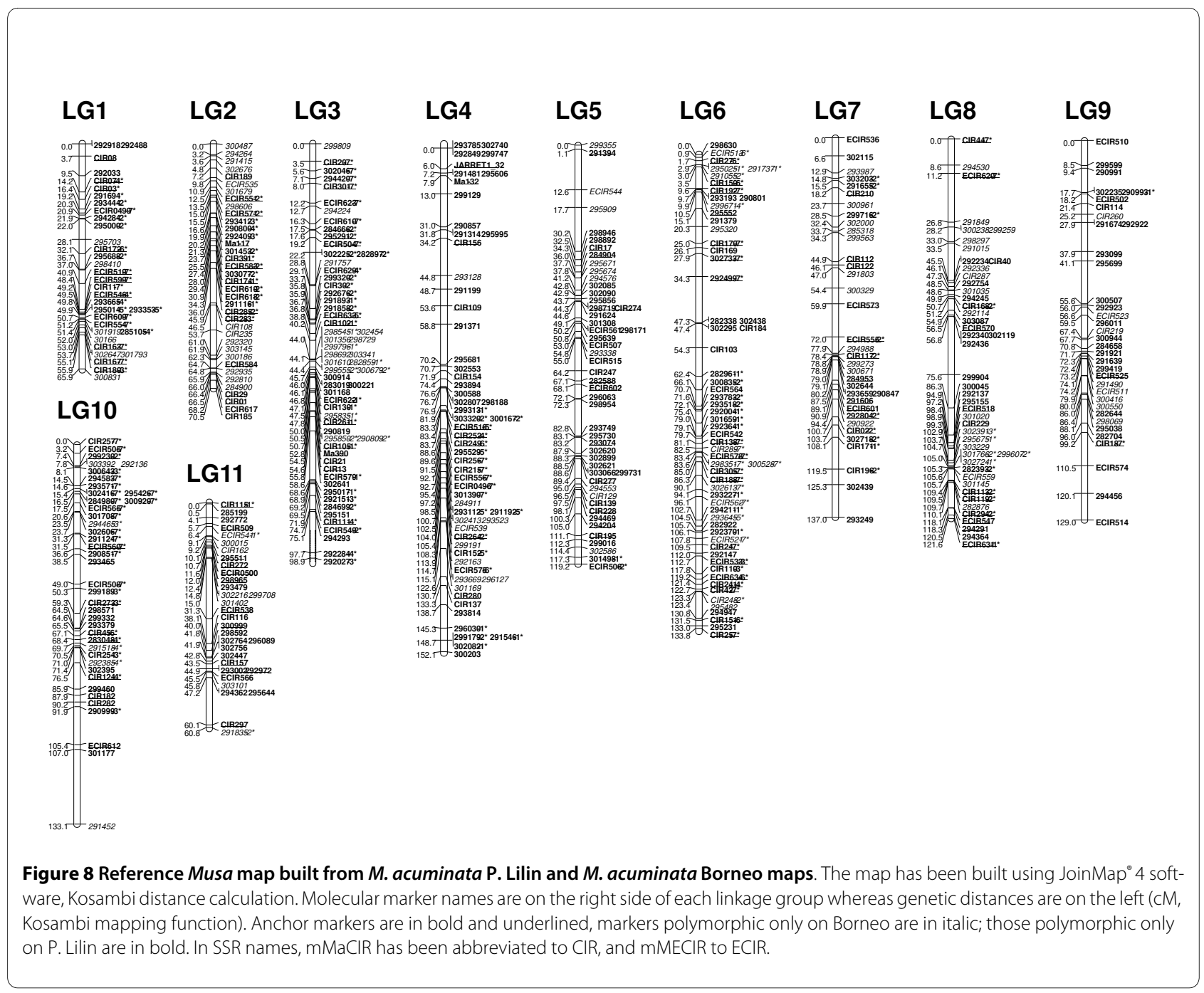

[31], among the parents of an $\mathrm{F}_{2}$ mapping population 'Calcutta 4' and 'Madang' [7] in order to physically assess the nature of the rearrangements. Based on our results, the investigations should focus first some of the linkage groups highlighted above: PLG1, PLG4, BLG 6, BLG 7 and BLG 8 . This is possible with the availability of several BAC libraries [32,33].

In our effort to produce a saturated map of Musa, we initially meant to study a progeny involving one structurally homozygous parent. The unexpected structural heterozygosity of Borneo induced additional complexity; yet it is likely that the rearrangement patterns involved different chromosomes and did not overlap in the two parents. Thus, we think our synthetic map can be a valuable reference for a $M$. acuminata genetic map. Whether this map corresponds to a "standard structure" [5] representative of the ancestral state requires confirmation with other mapping studies involving other germplasm compartments. The finding of structural heterozygosity in the "wild" Borneo challenges the idea that the wild forms have developed several genome arrangements in distinct populations, identified as subspecies, which then cause sterility and favor domestication among hybrids. Borneo belongs to the Microcarpa subspecies and has been found recently [34] to include some Bansksii subsp. alleles, indicating probably an intersubspecific origin. The structural heterozygosity in this case should be sufficiently limited to not affect fertility.

It may be difficult to establish the ancestral configuration by comparing only A genome forms; the use of a B genome representative as an outgroup will certainly be helpful.

The case of the relation between linkage groups 1 and 4 in P. Lilin sheds light on particular aspects of structural polymorphisms in banana. Our current interpretation is the duplication of a segment featuring at least fifteen markers, including eight SSR loci, even if the breakpoint on PLG1 should be refined more precisely. Coding the markers/alleles as co-dominant (forcing a single locus) or as dominant (allowing two loci) in the segregation data 
does not modify the global size of the segment: these markers span on about $1 \mathrm{cM}$ in P. Lilin. In contrast, they span on about $15 \mathrm{cM}$ in Borneo.

If the cause for the distortions and the pseudolinkages is indeed this duplication, it implies that P. Lilin has three copies of the loci involved in the duplication. Yet no case was found where one of these loci displayed three distinct alleles, meaning that the duplication features a haplotype that is conserved along a segment which spans $15 \mathrm{cM}$ in the Borneo configuration. This would mean that this duplication is recent enough for bearing no trace of SSR mutation nor of recombination with other haplotypes. P. Lilin is clearly incompletely fertile, but it was sufficiently fertile to produce the large number of progeny that we analyzed. We cannot exclude that this configuration is unique to P. Lilin, neither can we exclude it from a wider group of wild accessions that exchange genetic material. This leads to the question of the population dynamics of structural heterozygosity: if a heterozygous configuration leads to genetic map constrictions, and if it does not hamper reproduction, a fraction of the progeny that is again heterozygous should display the same genetic constriction. This could induce specific linkage disequilibrium in the area of rearrangement. Therefore, it can be worth testing whether this type of evidence, namely linkage disequilibrium among stretches of markers, can help infer the distribution of structural rearrangements in the whole species.

As a more direct route, strong segregation distortions induced by structural rearrangements can also be monitored using small-sized progenies (30-50 individuals) and be subjected to the neighbor joining approach that we have used.

\section{Conclusions}

We present here the first dense genetic map of $M$. acuminata with the expected eleven linkage groups, on the basis of a synthesis between two parental maps featuring distinct patterns of segregation distortions. This map can serve as a tentative reference for further studies. It displays 167 SSR and 322 DArT loci, covering $1197 \mathrm{cM}$ with an average density of one marker for $2.8 \mathrm{cM}$.

It will be central in further analysis of the genome of Musa. Current projects plan a complete sequencing of the A genome of a particular doubled haploid derived from Pahang, a Malaccensis genotype. In that project, density of the genetic map will be further increased.

This synthetic map is also accompanied with hypotheses for structural rearrangements and selection pressures which occur in the two parents, with more precision in $\mathrm{P}$. Lilin. Our understanding of these rearrangements is not complete, but our findings do provide testable hypotheses for molecular cytogenetic studies for visualizing structural polymorphisms. It is clear that more genome structure analyses are needed for understanding the patterns in the A genome, which are very important for breeding activities. The SSR markers provide a framework for selecting polymorphic markers for new mapping studies, whereas the DArT markers will efficiently complement a loose SSR selection for efficiently filling potential gaps. Therefore, new maps can be quickly generated when new progenies are available. A complementary ad hoc effort could also be needed to develop co-dominant markers which can reveal dosage effect in order to help resolve patterns which involve structural rearrangements.

\section{Methods \\ Material}

The segregating population, named "Borli" in this paper, was obtained from the intraspecific cross of two heterozygous diploid accessions [M. acuminata 'Borneo' x M. acuminata cv. 'Pisang Lilin']. The parents were chosen based on a combination of different criteria such as genetic distance $(0.8$ from simple matching distance based on 20 SSR markers, data not shown), allelic heterozygosity ( $>60 \%$ for each parent on the basis of 20 SSR study, data not shown) and putative structural heterozygosity. The M. acuminata 'Borneo' female parent belongs to subsp. microcarpa and is a "wild" seeded accession originated from Indonesia. It was shown as a structural homozygote belonging to the "standard group" [5]. The $M$. acuminata 'P. Lilin' variety is a male fertile $M$. acuminata subsp. malaccensis derivative originating from Malaysia, and supposedly heterozygous for one translocation as a hybrid from Central and North Malayan translocation groups $[5,10]$.

The cross was made in Guadeloupe (French West Indies). The $\mathrm{F}_{1}$ hybrids were germinated in vitro [35] and the 268 plantlets then transferred to a greenhouse in Montpellier. The ploidy level of all individuals was assessed by flow cytometry and chromosome counts; one case of triploidy was observed and the corresponding individual was removed. One hundred and eighty randomly chosen individuals were genotyped for segregation analysis.

\section{DNA isolation}

Leaves were harvested for DNA extraction 2 months after plant transfer to the greenhouse. $3 \mathrm{~g}$ of frozen material was ground with liquid nitrogen with a mortar and pestle following the modified Matab method [36]. The DNA was re-suspended in PCR grade water after isopropanol evaporation.

\section{Ploidy level}

As polyploid or aneuploid individuals are often observed in Musa progenies [37,38], the ploidy level of the whole progeny (i.e. 268 plants) was determined by flow cytome- 
try. Nucleus extraction and staining were prepared with a CyStain UV ploidy kit (Partec CyStain UV ploidy kit, Partec GmbH, Münster, Germany). The analyses were performed with a PAS flow cytometer (Partec) equipped with an HBO lamp for UV excitation. A leaf sample of each individual plant was chopped together with leaf tissue of $M$. acuminata $\mathrm{cv}$. Cavendish clone $(2 \mathrm{~N}=3 \times=33)$ as an internal reference. Each plant underwent two independent measurements.

As a cross reference, chromosome counts on root tip squashes [39] were also performed on a sample of 20 individuals.

\section{Cytogenetic analysis}

Immature anthers were excised from male flower buds of both Borneo and P. Lilin clones from 28 to 33 days before anthesis, and fixed overnight in a 1:3 acetic acid: ethanol solution saturated with ferric acetate. They were then transferred into $70 \%$ ethanol and stored at $8^{\circ} \mathrm{C}$ in this solution.

Meiotic chromosomes were studied in pollen mother cells (PMCs) squashed in aceto-carmine staining solution. Observations were performed on metaphases I containing enough well flattened cells to complete brightfield analysis at $\times 1000$ magnification or differential interferential contrast analysis. An average of 15 PMCs per clone were scored for chromosome rearrangements.

\section{SSR markers}

We tested 38 SSR markers from a SSR enriched library constructed from M. acuminata 'Gobusik' (mMaCir01 to $\mathrm{mMaCir} 45$ [40], Jarret 03 to Jarret 1_32 and Ma1_17 to Ma 3_90 [41] and 205 SSR markers from another SSR enriched library for (CA)n and (CT)n microsatellite and constructed from $M$. acuminata 'Calcutta 4' and M. balbisiana 'Pisang Klutuk Wulung' (mMaCir101-EMBL AM950326 to mMaCir307 -EMBL AM950533). The SSR markers with their primer pairs are presented in Table 2. We also used 143 markers from a M. acuminata 'Calcutta 4' EST library (mMECir501-EMBL $\underline{\text { FM878660 }}$ to mMeCir642-EMBL78794). The other SSR markers came from other EST sequences (mMECIR491 to mMECir500; Table 2 and [42]) from M. acuminata "Cavendish".

For a given SSR locus, the forward primer was designed with a 5'-end M13 extension (5'-CACGACGTTGTAAAACGAC-3') in order to generate fluorescent amplicons after fluorescent dye hybridization. Primer pair tests were performed on a 96 well PTC 100 thermocycler using the same protocol and reaction mixture as described below, in a final volume of $20 \mu \mathrm{l}$.

The PCR amplification was performed in a 384 well Eppendorf mastercycler with 10 ng of Musa DNA in a 10 $\mu \mathrm{l}$ final volume of buffer (10 mM Tris- $\mathrm{HCl}(\mathrm{pH} 8), 100$
$\mathrm{mM} \mathrm{KCl}, 0.05 \% \mathrm{w} / \mathrm{v}$ gelatin, and $2.0 \mathrm{mM} \mathrm{MgCl} 2)$ containing $0.08 \mu \mathrm{M}$ of the M13-labelled primer, $0.1 \mu \mathrm{M}$ of the other primer, $160 \mu \mathrm{M}$ of $\mathrm{dNTP}, 1 \mathrm{U}$ of Taq DNA polymerase (Life Technologies, U.S.A.) and $0.06 \mu \mathrm{M}$ of M13 primer-fluorescent dye IR700 or IR800 (Biolegio, Netherlands).

The SSRs were amplified by couples. Two SSR markers were amplified at the same time in a 384 well plate under touchdown PCR conditions: initial denaturation at $94^{\circ} \mathrm{C}$ for $60 \mathrm{~s}$; touchdown cycles were performed at a rate of $1^{\circ} \mathrm{C} /$ cycle. The first cycle was conducted at the highest primer melting temperature (TM), and the last cycle of the touchdown program was conducted at the lowest primer pair TM. These initial cycles were followed by 35 cycles at $94^{\circ} \mathrm{C}$ for $30 \mathrm{~s}$, [lowest $\mathrm{TM}-1^{\circ} \mathrm{C}$ ] for $60 \mathrm{~s}$, and $72^{\circ} \mathrm{C}$ for $120 \mathrm{~s}$; and a final elongation step at $72^{\circ} \mathrm{C}$ for 5 min. For example, if the TM of the first pair was $58^{\circ} \mathrm{C}$ and the TM of the second pair was $53^{\circ} \mathrm{C}$, we performed the first cycle at $59^{\circ} \mathrm{C}$; the 7 following cycles were performed in touchdown at $53^{\circ} \mathrm{C}$. PCR was achieved with 35 more cycles at $52^{\circ} \mathrm{C}$.

IR700 or IR800-labeled PCR products were diluted 8fold and 5-fold respectively, subjected to electrophoresis in a $6.5 \%$ polyacrylamide gel and then sized by the IR fluorescence scanning system of the sequencer. For each run, a ladder (range 71 to $367 \mathrm{bp}$ ) was added at the edges of the gel. The gel pictures were analyzed using AFLPquantar Pro software [43] with two independent readings.

\section{DArT markers}

DArT arrays were produced from individualized clones of libraries prepared from PstI-based genomic representations [44]. Two genomic representations comprising 11520 DArT markers were generated from 53 Musa genotypes ([45]). A random sample of 92 plants, out of the 180 analyzed with SSRs, was analyzed on each representation. The DArT markers heterozygous on one parent only were scored as co-dominant (segregation ratio 1:1), whereas the DArT markers polymorphic on both parents were scored as dominant (segregation ratio 1:3).

\section{Segregation distortion}

As a diagnostic test for marker reliability, all markers were tested for significant deviation from expected Mendelian segregation ratios using a chi-square test prior to linkage. Markers that deviated significantly from Mendelian ratios were re-checked for scoring errors. Distorted markers are denoted on the map representations with asterisks according to their distortion level (i.e. $1^{*} \mathrm{P}<$ 0.05, 2* $\mathrm{P}<0.01,3^{*} \mathrm{P}<0.005,4^{*} \mathrm{P}<0.001,5^{*} \mathrm{P}<0.0005$, $\left.6^{*} \mathrm{P}<0.0001,7^{*} \mathrm{P}<0.00005\right)$. 
Table 2: Characteristics of the mapped SSR loci from a Musa genomic library (mMaCIR102 to mMaCIR 305) and from EST sequences (mMECIR0494 to mMECIR0500).

\begin{tabular}{|c|c|c|c|c|c|c|c|}
\hline SSR & EMBL & motif & LG & forward primer & reverse primer & Ann $\left({ }^{\circ} \mathrm{C}\right)$ & Size (bp) \\
\hline$\overline{\mathrm{mMaCIR} 102}$ & AM950328 & (AG) $10,(\mathrm{TG}) 5$ & 6 & TGTTGGATTGGCTTCATC & CTTCGTTCAATGGTCTCCT & 55 & 220 \\
\hline $\mathrm{mMaCIR} 103$ & AM950329 & (CT) 14, & 3 & ССTCTTCTCCCTGTGTTG & CGGTTAAACATACCTATTCTTG & 54 & 179 \\
\hline mMaCIR105 & AM950331 & (CA) $8,(C T) 15$ & 6 & CATCCACTTGCTTTTCCA & CTTCACGGCTTCCACA & 56 & 264 \\
\hline $\mathrm{mMaCIR} 108$ & $\underline{\text { AM950334 }}$ & (CA)7,(CA)4, & 2 & ACGCATGGTAAAGTGGAA & ACATTCAAATCACGTTGCT & 55 & 111 \\
\hline mMaCIR109 & $\underline{\text { AM950335 }}$ & (CA) 13, & 4 & ACTCTAGTTCCAGAATAACTCCA & CAATCTTCATTAGCCAGTTGT & 55 & 204 \\
\hline $\mathrm{mMaCIR} 110$ & $\underline{\mathrm{AM} 950336}$ & $(\mathrm{AC}) 7,(\mathrm{GA}) 6$ & 3 & GGTGAACTGATGTGCGA & TCTTTCAACGGAATAAGCA & 55 & 244 \\
\hline $\mathrm{mMaCIR} 111$ & $\underline{\mathrm{AM} 950337}$ & (CA)8, & 6 & TCGTATGGAACAACAGTCC & CTITCACCTTCAAACAGCA & 55 & 137 \\
\hline $\mathrm{mMaCIR} 112$ & AM950338 & (CA) $5,(C A) 15$ & 7 & GTTCGGCTGGAGGTAGTT & AAGAACACGAAGGCAGG & 55 & 330 \\
\hline $\mathrm{mMaCIR} 113$ & $\underline{\text { AM950339 }}$ & (CA) 10, & 10 & TCAAGTATTTCACCGTATTGC & TTACCACCCTGTCATCTTTC & 55 & 207 \\
\hline $\mathrm{mMaCIR} 114$ & $\underline{\mathrm{AM} 950340}$ & $(\mathrm{AC}) 7,(\mathrm{CT}) 28$ & 8 & GCAAGCCAAAGGGAA & ACCAACAAAGAATGGTGTAA & 54 & 222 \\
\hline $\mathrm{mMaCIR} 115$ & AM950341 & (CA)2, & 11 & CAAGAGACTACCACCGAAGA & TGATTCTCACGACGTATGG & 55 & 114 \\
\hline $\mathrm{mMaCIR} 116$ & AM950342 & (TC)2,(TC)20 & 11 & ACACAAAGAAACCAGCCA & CGTCCCATCGTCTCCT & 55 & 202 \\
\hline $\mathrm{mMaCIR} 117$ & AM950343 & (TC)20, & 7 & GTTGTGGAATAAGTGGGAA & ATGAGGGAGTTAGTGGTGG & 55 & 214 \\
\hline $\mathrm{mMaCIR} 119$ & $\underline{\mathrm{AM} 950345}$ & (CA)9,(TA)6,(CA)5, & 10 & TGAAAAGCAATCCAACCT & ACCCTGAAATGTTTGTCTTT & 54 & 395 \\
\hline $\mathrm{mMaCIR} 122$ & AM950348 & (GT)8, & 7 & CGGTGACACTGGAAGGT & CAACTGAAGAACTGCCACTAA & 56 & 204 \\
\hline $\mathrm{mMaCIR} 124$ & AM950350 & $(\mathrm{AC}) 7$ & 9 & ACCTTGACAGCCCTCTTC & ATCAATCATTTCTGGGGTT & 55 & 63 \\
\hline $\mathrm{mMaCIR} 129$ & AM950355 & (CA)6, & 5 & CTAACCTITGATTCTGTITG & GTCCCTGATACACCATTC & 50 & 214 \\
\hline $\mathrm{mMaCIR} 130$ & AM950356 & (TG) 17, & 6 & TTAAACGTCTCCGTGTCTTC & TTGCATGAGGCTGGG & 56 & 311 \\
\hline $\mathrm{mMaCIR} 137$ & AM950363 & (TC)12, & 4 & CGTATTCTACATCTGCTTCTTT & GCAGTGATTAGGTGATGATTT & 54 & 223 \\
\hline $\mathrm{mMaCIR} 138$ & AM950364 & (CA)7, & 3 & TCATTCTCATGCGGAACT & CGGTGGATGTTGTTGG & 55 & 173 \\
\hline mMaCIR139 & $\underline{A M 950365}$ & (GA) 18, & 5 & TCGTCCCCTACTGCCT & ATGCTTCCGTTTGGCT & 55 & 187 \\
\hline $\mathrm{mMaCIR} 150$ & AM950440 & $(\mathrm{CA}) 10$ & 3 & ATGCTGTCATTGCCTTGT & GAATGCTGATACCTCTTTGG & 55 & 238 \\
\hline $\mathrm{mMaCIR} 151$ & AM950441 & $(\mathrm{CT}) 21$ & 3 & TATCCACCTCCTGGCAC & GCCAAACATCACCCAAC & 55 & 172 \\
\hline $\mathrm{mMaCIR} 152$ & AM950442 & (CTT) 18,(CT)17,(CA)6 & 4 & CCACCTTTGAGTTCTCTCC & TTTCCCTCTTCGATTCTGT & 55 & 163 \\
\hline mMaCIR154 & AM950444 & (CT) 17 & 4 & CATTCAGCATGGAAACCT & СТTCCTCAAACTGCTCCTC & 55 & 311 \\
\hline $\mathrm{mMaCIR} 156$ & AM950446 & (TG) 23 & 4 & CTTTCTGAAGGAAATTCTGAC & AGTGCAGCCCAATGAA & 54 & 210 \\
\hline $\mathrm{mMaCIR} 157$ & AM950447 & (CA)9,(TA)7 & 11 & TGGTATTATTTCATAGCCCTTC & ATGGTATTGTTGGATGGTGT & 55 & 272 \\
\hline $\mathrm{mMaCIR} 162$ & AM950452 & (CA) 8 & 11 & CTGCCTGTCCCACGA & GCGGCCATCATAATTCC & 57 & 161 \\
\hline $\mathrm{mMaCIR} 163$ & AM950453 & (AC) 14 & 1 & TGAAACAATCTTCATCAGCT & TCTGGACTTGGATGCTATTT & 55 & 247 \\
\hline $\mathrm{mMaCIR} 167$ & AM950367 & $(\mathrm{AC}) 7$ & 1 & САCTTCCACCTCTGCATC & GGTCTACTAACTTGAACACGAAC & 55 & 336 \\
\hline $\mathrm{mMaCIR} 168$ & AM950368 & (CA)7, & 10 & GCACCAAACCAGTCCTAC & CGTCTCAGTTGCCGTG & 55 & 243 \\
\hline mMaCIR169 & $\underline{\text { AM950369 }}$ & $(\mathrm{CT}) 14,(\mathrm{CA}) 1$ & 3 & TTTGGAGGAGACCATGATT & GCATTACATATCCTGCCTTT & 55 & 297 \\
\hline $\mathrm{mMaCIR} 170$ & $\underline{\mathrm{AM} 950370}$ & (CA) 8 & 3 & GGGCCTCCATAAGCAA & АCTTACCTTCCTGCCCAC & 55 & 202 \\
\hline $\mathrm{mMaCIR} 171$ & AM950371 & (CA) $5,(\mathrm{GA}) 10$ & 7 & GTAATACAAGTCTTCAGAGCAT & CTGTTTCGCCACTATCTT & 51 & 192 \\
\hline $\mathrm{mMaCIR} 172$ & AM950372 & (CT)19, & 1 & CAGCTAATGCCAAACCC & CGACTTCGAGCGAGC & 55 & 258 \\
\hline $\mathrm{mMaCIR} 174$ & AM950374 & (AG)13, & 2 & GAACCCACCTCCCTCTT & TGGGATTCCTGAGTGCT & 55 & 167 \\
\hline $\mathrm{mMaCIR} 180$ & AM950380 & (CA)7, & 1 & GCCTCAGCCTCATCATC & CACCCACTCGACCCA & 55 & 226 \\
\hline $\mathrm{mMaCIR} 182$ & AM950382 & (TC)22, & 9 & AACGCTTCTGCCTTGTT & TGAGACGTATTGCCCTAGTT & 55 & 150 \\
\hline $\mathrm{mMaCIR} 184$ & AM950384 & (TG)7, & 3 & TGTCATCGGCATAGACTG & TGGAATTGAACTGAAGCC & 54 & 314 \\
\hline $\mathrm{mMaCIR} 185$ & AM950385 & (TG)8, & 2 & САПTTCTATTCCCAGTCCC & CCAATGTTACTTCCATGCT & 54 & 181 \\
\hline $\mathrm{mMaCIR} 188$ & AM950388 & (TG)9,(TA)7, & 3 & GTGCTTGTTCGCTTGTIT & AGCCCAAGTATCCCACC & 56 & 160 \\
\hline mMaCIR189 & AM950389 & (CT)3,(CT)16 & 2 & GGGAGGGCAGAGGAA & GCCGAACTTGGTAATGTG & 55 & 259 \\
\hline mMaCIR192 & AM950458 & (TG)8 & 3 & TGACCTAGCACAACGCA & GCTTATGTTTCATCGCCTT & 56 & 133 \\
\hline mMaCIR193 & AM950459 & (AC)8 & 9 & TGTCCCTATCTGTCCTCTTT & CGCTTTGGAGTGTGCT & 54 & 301 \\
\hline $\mathrm{mMaCIR} 195$ & AM950461 & $(\mathrm{GA}) 11,(\mathrm{GA}) 6$ & 5 & GAATCGCCTTAGTCTCACC & TCATGTGCTCCCATCTTT & 55 & 285 \\
\hline
\end{tabular}


Table 2: Characteristics of the mapped SSR loci from a Musa genomic library (mMaCIR102 to mMaCIR 305) and from EST sequences (mMECIR0494 to mMECIR0500). (Continued)

\begin{tabular}{|c|c|c|c|c|c|c|c|}
\hline mMaCIR196 & AM950462 & $(\mathrm{TA}) 4,(\mathrm{TC}) 17,(\mathrm{TC}) 3$ & 7 & GCTCCAAACCTCCCTIT & CGATGCCACACTGGAC & 55 & 173 \\
\hline $\mathrm{mMaCIR} 210$ & $\underline{\text { AM950476 }}$ & $(\mathrm{GA}) 3,(\mathrm{TG}) 12,(\mathrm{AG}) 5$ & 7 & GGAAGGTGGCATGAAAG & TAACCTGATACCCATGTATTGA & 55 & 319 \\
\hline $\mathrm{mMaCIR} 214$ & $\underline{\mathrm{AM} 950480}$ & $(A C) 7$ & 10 & CCATTGAGAGATCAACCC & CTATITGACGTTGGTGGTC & 54 & 107 \\
\hline $\mathrm{mMaCIR} 215$ & $\underline{\mathrm{AM} 950481}$ & $(\mathrm{GT}) 7,(\mathrm{AT}) 3$ & 4 & AAGTTGGAGATATAGAATGGGT & TCCAGTGAATATGGATCAGT & 54 & 327 \\
\hline $\mathrm{mMaCIR} 219$ & $\underline{\mathrm{AM} 950485}$ & $(\mathrm{GA}) 18,(\mathrm{AC}) 1$ & 8 & GGGTAAGCTCAAGATGGAA & CAGACGCTAAACGACACC & 55 & 320 \\
\hline $\mathrm{mMaCIR} 228$ & $\underline{\text { AM950494 }}$ & (CT) 18,(AC)7 & 5 & CAAGCATGTTAGTITGGGA & AAGGTGCATCCAAGGG & 55 & 197 \\
\hline $\mathrm{mMaCIR} 229$ & $\underline{\mathrm{AM} 950495}$ & $(\mathrm{GA}) 22$ & 10 & CTGGGTTCCTCACCTTCT & GAAACACCATGTCCCAAA & 55 & 253 \\
\hline $\mathrm{mMaCIR} 235$ & $\underline{A M 950501}$ & $(\mathrm{CT}) 7,(\mathrm{CT}) 8$ & 2 & CCATCCCAGGCCATA & GCCCAGAGTCCGAAAG & 55 & 329 \\
\hline $\mathrm{mMaCIR} 241$ & $\underline{\mathrm{AM} 950503}$ & $(\mathrm{TC}) 20$ & 3 & GCTAAGCATCAAGTAGCCC & ACGAACAAGCAATCAAAGTAG & 55 & 297 \\
\hline $\mathrm{mMaCIR} 247$ & $\underline{\text { AM950395 }}$ & (GT)10, & 5 & AATGGATTGGGCATCAG & GGAGGGAGGAGGGTTT & 55 & 178 \\
\hline $\mathrm{mMaCIR} 248$ & AM950509 & (TG)6,(GA)6,(AG)8 & 3 & ATGCCTGCTACCACCTC & GCAGTTCCACAGTCCAAG & 55 & 251 \\
\hline $\mathrm{mMaCIR} 249$ & AM950396 & (TG)9, & 4 & TGTATTGTATCCCTAATGTCCC & CCTTACTAGCCAATTACGTGAG & 56 & 279 \\
\hline $\mathrm{mMaCIR} 252$ & AM950510 & (TC)9,(TC)3 & 4 & TCGTAAGCGAAAGGTCG & CGAACGCACTACCACTATG & 56 & 180 \\
\hline $\mathrm{mMaCIR} 254$ & AM950512 & (CT) 25 & 9 & CATGGAGGGTTAGGAGC & ATGCTTATTCTATGGTGGTTG & 54 & 180 \\
\hline $\mathrm{mMaCIR} 256$ & $\underline{\text { AM950399 }}$ & (CA)7, & 4 & TTGCGGGAAACTGCT & GTTGCACTGCCCACTT & 54 & 280 \\
\hline $\mathrm{mMaCIR} 257$ & $\underline{\mathrm{AM} 950400}$ & $(\mathrm{CA}) 7$ & 9 & CTITACCGAGTTGAGGG & TCATATCAGAAGATAGCCAA & 51 & 234 \\
\hline $\mathrm{mMaCIR} 260$ & $\underline{\mathrm{AM} 950515}$ & (TG)8 & 8 & GATGTITGGGCTGTITCT & AAGCAGGTCAGATTGTTCC & 55 & 189 \\
\hline $\mathrm{mMaCIR} 261$ & $\underline{\mathrm{AM} 950516}$ & (CA)13 & 6 & TATCAGGCATACGTTCTGTAG & AAAGAAGGTGGGTGATAGG & 54 & 202 \\
\hline $\mathrm{mMaCIR} 264$ & $\underline{A M 950519}$ & (CT) 17 & 4 & AGGAGTGGGAGCCTATTT & CTCCTCGGTCAGTCCTC & 54 & 235 \\
\hline $\mathrm{mMaCIR} 272$ & $\underline{A M 950408}$ & $(\mathrm{AC}) 6,(\mathrm{CT}) 5$ & 11 & CTCACCGGATGGCAC & GGCATTAAGTITCAGGAATAAG & 55 & 171 \\
\hline $\mathrm{mMaCIR} 273$ & $\underline{A M 950521}$ & (TC)22,(CT)6 & 9 & TGGTTGAAGATTCCCAT & GATCAAGAGGTGACAAACC & 53 & 211 \\
\hline $\mathrm{mMaCIR} 274$ & $\underline{\text { AM950409 }}$ & (AC)11, & 5 & TAGCTCTITCAACACTCTCATC & CTGGAGGCAGCGAAC & 54 & 150 \\
\hline $\mathrm{mMaCIR} 277$ & $\underline{\mathrm{AM} 950523}$ & (TG) 12 & 5 & ACGATAGGATTATTGGCTGT & GGCTCTTAATITGACAAGAA & 54 & 212 \\
\hline $\mathrm{mMaCIR} 280$ & $\underline{A M 950412}$ & $(\mathrm{TC}) 7,(\mathrm{AC}) 7$ & 4 & GGGTCCCTGTTGGCT & TTGCAGATTAGGGTGGG & 55 & 221 \\
\hline $\mathrm{mMaCIR} 282$ & AM950414 & $(\mathrm{AG}) 8,(\mathrm{AG}) 3,(\mathrm{TG})$ & 9 & CATCCTGTTGCTCCCTC & AAGAATCTAGCAGCATCCAA & 55 & 209 \\
\hline $\mathrm{mMaCIR} 285$ & AM950416 & $(\mathrm{TC}) 21,(\mathrm{AC}) 7,(\mathrm{AC}) 5$ & 2 & ATTGCCATGATTGACCC & TACGGCTCCTATCGTCC & 55 & 183 \\
\hline $\mathrm{mMaCIR} 287$ & $\underline{A M 950526}$ & $(\mathrm{TG}) 7$ & 10 & TITAAGAATCCCTCGCTTT & ACAGATGACGAACAAACTACC & 54 & 203 \\
\hline $\mathrm{mMaCIR} 289$ & $\underline{\mathrm{AM} 950418}$ & (GT)8,(TC)3, & 3 & TTGCTTCCTGTAACATCTCC & GGTCTGGGTGAAGGCA & 56 & 205 \\
\hline $\mathrm{mMaCIR} 294$ & $\underline{A M 950421}$ & $(\mathrm{AC}) 7,(\mathrm{TC}) 8,(\mathrm{CA}) 5$ & 10 & CACGAGTCATAATCCAGTCA & GTTCAAAGCTCGTTGGG & 55 & 178 \\
\hline mMaCIR297 & $\underline{A M 950424}$ & $(\mathrm{TC}) 9,(\mathrm{AC}) 13,(\mathrm{CA}) 9$ & 11 & GAACTCGGATTGTTCCTIT & AGGCTGATGGTAGCGAG & 55 & 173 \\
\hline mMaCIR301 & $\underline{A M 950427}$ & (TG)11， & 6 & CATGATGTTTGAGTITGC & CTGGAAAGCAACACCG & 54 & 166 \\
\hline $\mathrm{mMaCIR305}$ & $\underline{A M 950429}$ & $(\mathrm{CA}) 5,(\mathrm{TC}) 6,(\mathrm{GA}) 3$ & 3 & CCGATCAATTCAGCCA & TATGAGCAAGAACAGCCC & 55 & 299 \\
\hline mMECIR0494 & pCav22 & $(\mathrm{TG}) 6 \mathrm{c}(\mathrm{GT}) 12$ & 9 & CCATGATACGGGCTTACGA & TCAATTACCAGCATCCTTACTT & 56 & 266 \\
\hline mMECIR0496 & ATPVScl3 & $(\mathrm{TA}) 7$ & 4 & CGCCACATAAGGCTCCCT & GTCGCCATCTCCTTGAA & 55 & 176 \\
\hline mMECIR0498 & SSHBSVban9a08 & (GT)8nnnnn(TTC)13 & 6 & CGGGGTCGTGTCTTAGGAA & GCAATCACACGGATACCTC & 56 & 183 \\
\hline mMECIR0499 & SSHBSVban9a08 & (AG)8) & 1 & CGCTTGCCTTTGGTTGTG & CCAGTAGACGCCAATGC & 56 & 172 \\
\hline mMECIR0500 & SSH didier $\mathrm{Cl} 3$ & $(A G) 9$ & 11 & CCAGCAGACGCACACAAA & GCAACTGCAAATGAGGG & 56 & 150 \\
\hline
\end{tabular}

Calculated primer annealing temperature (Tm) and expected product size in reference variety (i.e. M. acuminata 'Calcutta 4 and $M$. balbisiana "Pisang Klutuk Wulung" for mMaCIR102 to mMaCIR305 and M. acuminata 'Cavendish' for mMECIR0494 to mMECIR0500). Expected size does not take into account the M13 tail (19 bp) added to each 5'end of forward primer. LG: linkage group; Ann: Annealing temperature used.

\section{Linkage map construction}

Borneo and P. Lilin parental maps were built from the analysis of marker segregation in the progeny. The P. Lilin map was further refined based on hypotheses of structural rearrangements. Finally the structure of each chromosome of structural heterozygous pairs was given on the basis of the structural pattern leading to a simulated NJ tree (see below) that fitted the observed one.
A third map, named the "synthetic map", is meant to represent the standard/ancestral Musa chromosomes without rearrangement events. It was designed in part by using consensus linkage groups of parental maps. For the remaining non-consensus groups, we kept as a skeleton the parental linkage group supposed to be free of any rearrangement. Then, markers of the other homolog parental linkage group were introduced one by one and 
mapped when they did not affect the previous order. The assessment of normal versus rearranged linkage group was essentially based on the absence of skewed markers, but also based on the linearity of a Neighbor-Joining (NJ) tree representation of the linkage groups (see below). The final reference map is an "artificial" map supposed to best represent the "standard" Musa genome.

The linkage groups of each parental map were defined using JoinMap 4 software with a "cross-pollinated" population type [46]. Segregating DArTs markers present in the two sharing parents (dominant marker segregation type) were discarded, as they were poorly informative and not numerous in our data. A range of LOD scores from 3 to 8 was tested. The map was built at LOD 3.5 for Borneo, LOD 5 for P. Lilin and LOD 5 for the "synthetic map" using the regression mapping algorithm with the default parameters (i.e. a recombination frequency $<0.4$, a mapping LOD score threshold of 1 and Kosambi mapping function [46]). When linkage groups of the parental maps displayed collinearity, consensus groups were established integrating all initial genotyping data of the progeny, with the default parameters. In case of discrepancy in the alignment of the two linkage groups, we imposed the marker order from one parent as a fixed one for the other one and vice-versa. If the fixed orders gave incoherent mapping (negative distances, high mean chi-square, suspect double recombinants...), each parental group was drawn up independently.

Group names were assigned according to a previous partial map [7] if common markers were found. New names were given otherwise.

\section{Graphic representation of linkage groups}

Genetic mapping relies on the strong assumption that the recombination frequencies observed in a segregating population are accurate estimators of the genetic distances between markers physically aligned along the chromosomes. Therefore, defining the linear locus order and the distances between loci is strictly equivalent to a geometrical problem of adjusting the observed dissimilarities (the recombination frequencies) to a linear distance.

However, in case of heterozygous structural rearrangement, the observed recombination frequencies are locally a derivative of two unknown recombination frequencies corresponding to two different linear orders. For the affected loci, the conventional mapping methods propose a consensus that has no real biological meaning and they fail to map these loci correctly. However, keeping the geometrical problem analogy offers a means to localize these rearrangements graphically (Seguin, personal communication). Relaxing the constraint of linearity, the observed dissimilarities are adjusted to a so-called additive distance which is the distance on a tree-like graph [47] where the rearrangements appear as branches grafted on the basal line of unaffected loci. A linear distance is a particular case of additive tree distance, so in absence of rearrangement, the exhibited solution will be the optimal linear order of the loci. Several methods are available for adjusting a dissimilarity to a tree distance such as the NJ tree method [48], which remains computationally efficient even for a very large number of loci. These methods are based on agglomerative algorithms that join at the first step two close loci and progressively aggregate the closest remaining loci. This ascendant procedure means that the graph is essentially determined by the smallest dissimilarities. So, the highest values, between remote loci, which are truncated to 0.5 by construction in case of genetic, mapping, do not really disturb the tree construction

Keys to read the generated trees are given in Figure 3 from simulated data. A first set of chromosomes is generated with a marker each $5 \mathrm{cM}$. A second set is derived from the first one but with a particular rearrangement event. The simulated recombination frequencies are the arithmetic means of the genetic distance in the two chromosome sets. The simulation presumes that each segment of chromosome is able to pair with its homolog regardless of the structures of the chromosomes.

Figure 3-A is constructed from data of homologous chromosomes displaying fully aligned markers. It is the "normal" expected feature for homologous chromosomes. As soon as some rearrangements occur, the tree representation generates different figures such as Figure 3-B obtained in case of inversion. A distal non-reciprocal translocation generates a typical Y image (Figure 3-C), while a distal reciprocal translocation generates a typical $\mathrm{X}$ - shaped tree (Figure 3-D).

We applied the Weighted Neighbor-Joining algorithm implemented in DARwin 5 software [49] for the graphic representation of all the parental linkage groups identified under JoinMap 4 . For each linkage group independently, we exported from JoinMap 4 the pair-wise recombination frequencies which were converted into map distances using the Kosambi mapping function. The distance matrix was then exported to DARwin5 for NJ tree calculation and for graphic representation of the linkage group.

\section{Additional material}

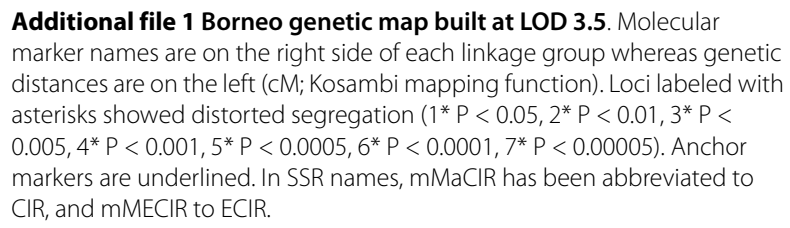




\section{Additional file 2 Pisang Lilin genetic map built at LOD 5. Molecular} marker names are on the right side of each linkage group whereas genetic distances are on the left (cM; Kosambi mapping function). Loci labeled with asterisks showed distorted segregation $\left(1^{*} P<0.05,2^{*} P<0.01,3^{*} P<\right.$ $\left.0.005,4^{*} P<0.001,5^{*} P<0.0005,6^{*} P<0.0001,7^{*} P<0.00005\right)$. Anchor markers are underlined. In SSR names, $\mathrm{mMaCIR}$ has been abbreviated to $\mathrm{CIR}$, and $\mathrm{MMECIR}$ to ECIR.

Additional file 3 Neighbor-joining tree designed from linkage group $6+8$ of Borneo defined at LOD 3.5; Markers in grey are DArTs markers, those in black SSR markers.

\section{Competing interests}

Andrzej Kilian (Director) and Eric Huttner are affiliated with Diversity Arrays Technology who provide DArT array commercial genotyping services for a range of crops.

\section{Authors' contributions}

IH participated in the conception and design of the study, genomic SSR primers design, SSR genotyping, $\mathrm{NJ}$ and mapping analysis and paper writing; $\mathrm{FB}$ in the conception and design of the study, embryo rescue, cytogenetics, chromosome counting, paper writing; MS in theoretical and methodological development of $\mathrm{NJ}$ tree applied to genetic mapping and paper reviewing, LG and RR in SSR genotyping, AMR in the development of the SSR enriched library, DArT array construction and paper reviewing; $\mathrm{CJ}$ in the creation of the population and design of the study, XP in the NJ analysis (theory and simulation), paper writing, FC in the development of the SSR enriched library, XA in EST SSR primers' design, IAK in DNA extraction and genotyping, RNGM in DNA extraction; PP in the EST library construction; GJP and TM in the EST library sequencing, DM in EST sequences generation, VB in the SSR enriched library sequencing, $\mathrm{EH}$ and $\mathrm{AK}$ in the DArT genotyping, $\mathrm{FCB}, \mathrm{AD}$ and $\mathrm{FXC}$ in the conception of the study, BC in mapping analysis and paper writing and JCG in the conception of the study and paper writing. All authors read and approved the final manuscript.

\section{Acknowledgements}

We thank the Generation Challenge Programme which commissioned the project and Bioversity International for coordinating the whole GCP project "Targeted Musa genome sequencing and frame map construction ». We also thank the Montpellier Languedoc-Roussillon Grand Plateau Technique Régional for hosting the SSR development and the SSR genotyping activities as well as the Grand Plateau Technique RIO Imaging for cytogenetical studies. We thank Claire Billot for her help on the genotyping platform and Christophe Périn for reviewing the manuscript.

\section{Author Details}

'CIRAD, UR Multiplication Végétative, Av. Agropolis, 34398 Montpellier Cedex 5, France, ${ }^{2}$ IIRAD, UMR DAP, Av. Agropolis, 34398 Montpellier Cedex 5, France, ${ }^{3}$ Current address: CIRAD, UMR Contrôle des maladies, Campus Baillarguet, 34398 Montpellier Cedex 5, France, ${ }^{4}$ CIRAD, UR Multiplication Végétative, Station de Neufchâteau, Sainte-Marie, 97130 Capesterre Belle-Eau, Guadeloupe FWI, ${ }^{5}$ Current address: CIRAD, UMR BGPI, Campus international de Baillarguet, 34398 Montpellier Cedex 5, France, ${ }^{6}$ Current address: Genomics Platform, Parco Tecnologico Padano, Via Einstein, Lodi, Italy, ${ }^{7}$ Nuclear Institute of Agriculture, Tando Jam, Sindh, Pakistan, 8 Instituto de Ciências Biológicas, Departamento de Biologia Celular, Universidade de Brasília, Campus Universitário Darcy Ribeiro, Asa Norte, CEP 70.910-900, Brasília, Brazil, 9Embrapa Recursos Genéticos e Biotecnologia, Brasília, Brazil, 10UMR QUALITROP, Station de Neufchâteau, Sainte-Marie, 97130 Capesterre Belle-Eau, Guadeloupe FWI, ${ }^{11 N I A S}$, Plant Genome Research Unit, Division of Genome and Biodiversity Research, 2-1-2, Kannondai, Tsukuba, Ibaraki 305-8602, Japan, 12CEA, DSV, IG, Genoscope, 2 rue Gaston Crémieux, 91000 Evry, France, ${ }^{13}$ Diversity Arrays Technology, PO Box 7141, Yarralumla, ACT 2600, Australia and ${ }^{14}$ CIRAD, UPR Systèmes bananes et ananas, Boulevard de la Lironde, 34398 Montpellier Cedex 5, France

Received: 18 September 2009 Accepted: 13 April 2010 Published: 13 April 2010

\section{References}

1. Loeillet D: Close-up: banana. FruiTrop 2008, 155(April):3-39.

2. Lescot T: Banana in figures - The world's favourite fruit. FruiTrop 2006, 140(December):5-9.

3. Bakry F, Carreel F, Jenny C, Horry J-P: Genetic Improvement of Banana. In Breeding Plantation Tree Crops: Tropical Species Edited by: Jain SM. Priyadarshan PM: Springer; 2009:3-51.

4. D'Hont A, Paget-Goy A, Escoute J, Carreel F: The interspecific genome structure of cultivated banana, Musa spp. revealed by genomic DNA in situ hybridization. TAG Theoretical and Applied Genetics 2000, 100(2):177-183.

5. Shepherd K: Cytogenetics of the genus Musa: Ipgri-Inibap. 1999:160

6. Fauré S, Noyer JL, Horry JP, Bakry F, Lanaud C, Goñzalez de León D: A molecular marker-based linkage map of diploid bananas (Musa acuminata). Theor App/ Genet 1993, 87(4):517-526.

7. Vilarhinos AD: Cartographie génétique et cytogénétique chez le bananier: caractérisation des translocations. Montpellier: Ecole Nationale Supérieure Agronomique; 2004.

8. Noyer JL, Dambier D, Lanaud C, Lagoda PLJ: The saturated map of diploid Banana (Musa acuminata). Plant and Animal Genome: january 1216 1997; San Diego 1997. Abstract P335

9. Udall JA, Quijada PA, Osborn TC: Detection of Chromosomal Rearrangements Derived From Homeologous Recombination in Four Mapping Populations of Brassica napus L. Genetics 2005, 169(2):967-979.

10. Dodds KS: Genetical and cytological studies of Musa. V. Certain edible diploids. Journal of Genetic 1943, 45:113-138.

11. Wilson GB: Cytological studies in the Musae. II. Meiosis in some diploid clones. Genetics 1946, 31:475-493.

12. Vilarinhos AD, Carreel F, Rodier M, Hippolyte I, Benabdelmouna A, Triaire D, Bakry F, Courtois B, D'Hont A: Characterization of translocations in banana by FISH of BAC clones anchored to a genetic map. Plant and Animal Genomes XIV San Diego 2006

13. Grapin A, Noyer J-L, Carreel F, Dambier D, Baurens F-C, Lanaud C, Lagoda PJL: Diploid Musa acuminata genetic diversity assayed with sequencetagged microsatellite sites. Electrophoresis 1998, 19(8-9):1374-1380.

14. Creste S, Tulmann Neto A, Vencovsky R, de Oliveira Silva S, Figueira A: Genetic diversity of Musa diploid and triploid accessions from the Brazilian banana breeding program estimated by microsatellite markers. Genetic Resources and Crop Evolution 2004, 51(7):723-733.

15. Creste S, Tulmann Neto A, de Oliveira Silva S, Figueira A: Genetic characterization of banana cultivars (Musa spp.) from Brazil using microsatellite markers. Euphytica 2003, 132(3):259-268.

16. Lorieux M, Goffinet B, Perrier X, León DG, Lanaud C: Maximum-likelihood models for mapping genetic markers showing segregation distortion. 1. Backcross populations. Theor App/ Genet 1995, 90(1):73-80.

17. Jáuregui B, de Vicente MC, Messeguer R, Felipe A, Bonnet A, Salesses G, Arús P: A reciprocal translocation between 'Garfi' almond and 'Nemared' peach. Theor App/ Genet 2001, 102(8):1169-1176.

18. Akhunov ED, Goodyear AW, Geng S, Qi L-L, Echalier B, Gill BS, Miftahudin, Gustafson JP, Lazo G, Chao S, et al:: The organization and rate of evolution of wheat genomes are correlated with recombination rates along chromosome arms. Genome Res 2003, 13(5):753-763.

19. Jarrell DC, Roose ML, Traugh SN, Kupper RS: A genetic map of citrus based on the segregation of isozymes and RFLPs in an intergeneric cross. Theor App/ Genet 1992, 84(1):49-56.

20. Kosterin O, Pukhnachev N, Gorel F, Berdnikov V: Location of the breakpoints of four reciprocal translocations involving group $\mathrm{V}$ and their influence on recombination distances between neighbouring markers. Pisum Genetics 1999, 31:13-20.

21. Semagn K, Bjørnstad $\AA$, Skinnes H, Marøy AG, Tarkegne Y, William M: Distribution of DArT, AFLP, and SSR markers in a genetic linkage map of a doubled-haploid hexaploid wheat population. Genome Res 2006, 49:545-555.

22. Wenzl P, Li H, Carling J, Zhou M, Raman H, Paul E, Hearnden P, Maier C, Xia $L$, Caig V, et al: : A high-density consensus map of barley linking DArT markers to SSR, RFLP and STS loci and agricultural traits. BMC Genomics 2006, 7(1):206.

23. Tadmor Y, Zamir D, Ladizinsky G: Genetic mapping of an ancient translocation in the genus Lens. Theor App/ Genet 1987, 73(6):883-892. 
24. Kianian SF, Quiros CF: Generation of a Brassica oleracea composite RFLP map: linkage arrangements among various populations and evolutionary implications. Theor App/ Genet 1992, 84(5):544-554

25. Barzen E, Mechelke W, Ritter E, Schulte-Kappert E, Salamini F: An extended map of the sugar beet genome containing RFLP and RAPD loci. Theor App/ Genet 1995, 90(2):189-193.

26. Quillet MC, Madjidian N, Griveau Y, Serieys H, Tersac M, Lorieux M, Bervillé A: Mapping genetic factors controlling pollen viability in an interspecific cross in Helianthus sect. Helianthus. Theor App/ Genet 1995, 91(8):1195-1202.

27. Ruiz C, Asins M: Comparison between Poncirus and Citrus genetic linkage maps. Theoretical and Applied Genetics 2003, 106(5):826-836.

28. Jenczewski E, Gherardi M, Bonnin I, Prosperi JM, Olivieri I, Huguet T: Insight on segregation distortions in two intraspecific crosses between annual species of Medicago (Leguminosae). Theor App/ Genet 1997, 94(5):682-691.

29. Pardo-Manuel de Villena F, Sapienza C: Nonrandom segregation during meiosis: the unfairness of females. Mamm Genome 2001, 12(5):331-339.

30. Dodds KS, Simmonds NW: Sterility and parthenocarpy in diploid hybrids of Musa. Heredity 1948, 2(1):101-117.

31. D'hont A: Unraveling the genome structure of polyploids using FISH and GISH; examples of sugarcane and banana. Cytogenetic and Genome Research 2005, 109:27-33.

32. Vilarinhos AD, Piffanelli P, Lagoda P, Thibivilliers S, Sabau X, Carreel F, D'Hont A: Construction and characterization of a bacterial artificial chromosome library of banana (Musa acuminata Colla). Theor Appl Genet 2003, 106(6):1102-1106

33. Šafář J, Noa-Carrazana JC, Vrána J, Bartoš J, Alkhimova O, Sabau X, Šimková H, Lheureux F, Caruana M-L, Doležel J, et al:: Creation of a BAC resource to study the structure and evolution of the banana (Musa balbisiana) genome. Genome 2004, 47(6):1182-1191.

34. Perrier X, Bakry F, Carreel F, Jenny C, Horry J-P, Lebot V, Hippolyte I: Combining biological approaches to shed light on the evolution of edible bananas. Ethnobotany Research and Applications 2009, 7:199-216.

35. Bakry F: Banana protocol - Zygotic embryo rescue. Fruits 2008, 63(2):111-115.

36. Risterucci AM, Grivet L, N'Goran JAK, Pieretti I, Flament MH, Lanaud C: A high-density linkage map of Theobroma cacao L. Theor App/ Genet 2000, 101(5):948-955

37. Dodds KS, Pittendrigh CS: Genetical and cytological studies of Musa. VII. Certain aspects of polyploidy. Journal of Genetics 1946, 47:162-177.

38. Dodds KS, Simmonds NW: Genetical and cytological studies of Musa. VIII. The formation of polyploid spores. Journal of Genetics 1946, 47(3):223-241

39. Bakry F, Shepherd K: Banana protocol - Chromosome count on banana root tips squashes. Fruits 2008, 63(3):179-181

40. Lagoda PJL, Noyer JL, Dambier D, Baurens F-C, Grapin A, Lanaud C: Sequence tagged microsatellite site (STMS) markers in the Musaceae. MolEcol 1998, 7(5):657-666.

41. Kaemmer D, Fischer D, Jarret RL, Baurens FC, Grapin A, Dambier D, Noyer $J$, Lanaud C, Kahl G, Lagoda PJL: Molecular breeding in the genus Musa: a strong case for STMS marker technology. Euphytica 1997, 96(1):49-63.

42. Mbéguié-A-Mbéguié $D$, Hubert $O$, Sabau X, Chillet M, Fils-Lycaon B, Baurens FC: Use of suppression subtractive hybridization approach to identify genes differentially expressed during early banana fruit development undergoing changes in ethylene responsiveness. Plant Science 2007, 172(5):1025-1036.

43. Geerlings H, Oeveren AJV, Pot JE, Schaik RCV: AFLP-QuantarTM Pro Image analysis software. 1999 [http://www.keygene-products.com]

44. Jaccoud D, Peng K, Feinstein D, Kilian A: Diversity Arrays: a solid state technology for sequence information independent genotyping. Nucleic Acid Research 2001, 29(4):e25

45. Risterucci A-M, Hippolyte I, Perrier X, Xia L, Caig V, Evers M, Huttner E, Kilian A, Glaszmann J-C: Development and assessment of Diversity Arrays Technology for high-throughput DNA analyses in Musa. Theor App Genet 2009, 119(6):1093-1103.

46. Van Ooijen JW: JoinMap 4 , software for the calculation of genetic linkage maps in experimental population. Wageningen, Netherlands: Kyazma B.V.; 2006

47. Buneman P: The recovery of trees from measures of dissimilarity. In Mathematics in Archeological and Historical Sciences Edited by: Hodson FR, Kendall DG, Tautu P. Edinburgh University Press; 1971:387-395.
48. Saitou N, Nei M: The Neighbor-Joining method: a new method for reconstructing phylogenetic trees. Mol Biol Evol 1987, 4(4):406-425.

49. Perrier $X$, Jacquemoud-Collet JP: DARwin software. 2006 [http:// darwin.cirad.fr/darwin/Home.php.

doi: 10.1186/1471-2229-10-65

Cite this article as: Hippolyte et al., A saturated SSR/DArT linkage map of Musa acuminata addressing genome rearrangements among bananas $B M C$ Plant Biology 2010, 10:65

\section{Submit your next manuscript to BioMed Central and take full advantage of:}

- Convenient online submission

- Thorough peer review

- No space constraints or color figure charges

- Immediate publication on acceptance

- Inclusion in PubMed, CAS, Scopus and Google Scholar

- Research which is freely available for redistribution

Submit your manuscript at www.biomedcentral.com/submit
C Biomed Central 